\title{
The Elementary Basic Principles of the Unified Theory of Relativity. $C_{1}$ Introduction* \\ VÁCLAV HLAVATÝ
}

\author{
Graduate Institute for Applied Mathematics, Indiana University, Bloomington, Indiana \\ Dedicated to Professor J. A. Schouten on his $70^{\text {th }}$ birthday
}

\section{CONTENTS}

Chapter I.

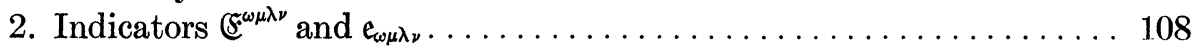

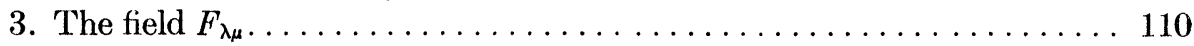

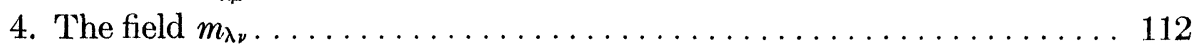

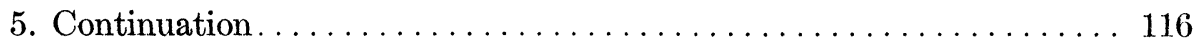

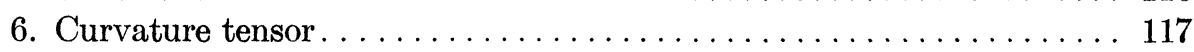

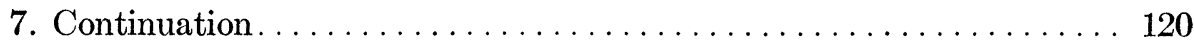

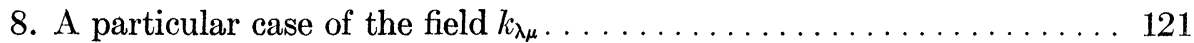

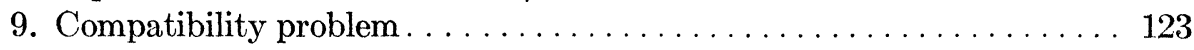

Chapter II.

1. Introduction . . . . . . . . . . . . . . . . . . . . . . . 128

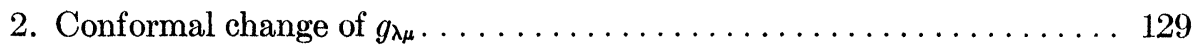

3. The most general solution $g_{\lambda \mu}$ of $(1.4) \ldots \ldots \ldots \ldots \ldots \ldots \ldots \ldots \ldots \ldots$

4. The most general solution $\Gamma_{\lambda \mu}^{\nu}$ of $(1.5) \ldots \ldots \ldots \ldots \ldots \ldots \ldots \ldots \ldots$

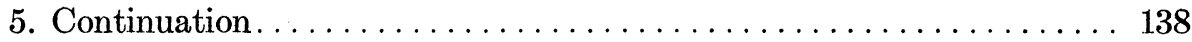

6. The most general solution of $(1.4)$ and $(1.5) \ldots \ldots \ldots \ldots \ldots \ldots$

7. Particular cases. . . . . . . . . . . . . . . . . . . . . . . 143

* Prepared under joint contract with the Office of Naval Research and the Army Office of Ordnance Research. 
The unified theory of relativity proposed by Einstein* is based on three principles:

A) The basic tensor $g_{\lambda \mu}$ is neither symmetric nor skew-symmetric.

B) It gives rise to a connection $\Gamma_{\lambda \mu}^{\nu}$ defined by

$$
\partial_{\omega} g_{\lambda \mu}=\Gamma_{\lambda \omega}^{\alpha} g_{\alpha \mu}+\Gamma_{\omega \mu}^{\alpha} g_{\lambda \alpha} .
$$

Whenever the tensor $g_{\lambda \mu}$ satisfies certain conditions, the system (I) admits only one solution $\Gamma_{\lambda \mu}^{\nu}$.

C) In order to get the arguments $g_{\lambda \mu}$ involved in $\Gamma_{\lambda \mu}^{\nu}$ one imposes a set of conditions which may be condensed into

$$
R_{\mu \lambda}=\frac{\partial}{\partial x}{ }_{[\mu} X_{\lambda]}
$$

Here $R_{\mu \lambda}$ is the contracted curvature tensor of the $\Gamma_{\lambda \mu}^{\nu}$, while $X_{\lambda}$ is an arbitrary vector.

The significance of the tensor $g_{\lambda \mu}$ has been dealt with in [5]. The connection $\Gamma_{\lambda \mu}^{\nu}$ mentioned in $B$ ) has been investigated in [6] and [7]. In this paper we shall deal with the system of partial differential equations mentioned in $C$ ). In dealing with this problem it has turned out to be advisable first to derive some fundamental properties of the systems mentioned in $B$ ) and $C$ ) and then to look afterward for physical application. In this present paper we confine ourselves to a thorough investigation of the aforementioned fundamental properties, which will be used in the next paper for physical applications.

The paper is divided into two chapters. In the first two sections of the first chapter we introduce the necessary prerequisites for a subsequent study of a skew-symmetric tensor $m_{\lambda_{\nu}}$ (Sections 3-5) which will play the role of the electromagnetic field in the next paper. Sections 6 and 7 deal with the fundamental properties of the curvature tensor which are necessary for the application of the system mentioned in $C$ ). In the eighth section we exhibit a tensor $g_{\lambda \mu}$ with special properties (needed in the next paper). In the last section of the first chapter a particular solution $g_{\lambda \mu}, \Gamma_{\lambda \mu}^{\nu}$ of the systems mentioned in $B$ ) and $C$ ) is given. In the second chapter we deal with conformal change of $g_{\lambda \mu}$ (Section 2), with affine symmetric transformation of $\Gamma_{\lambda \mu}^{\nu}$ (Section 5) and with existence problems (Sections 3, 4,6). Using a particular solution, we build up the most general solution of the systems mentioned in $B$ ) and $C$ ). The method devised for this purpose enables us also to pick out solutions which might be suitable for physical applications. The last section is devoted to a particular case which will be dealt with from the physical point of view in the first chapter of the next paper.

\footnotetext{
* See Appendix II in [1] and [2]. (Numbers in brackets refer to the bibliography at the
} end of the paper.) 


\section{Chapter I}

1. Auxiliary results. This section contains some known results taken from [5] and [6] (without proof, and in a slightly changed notation). These results will be needed in subsequent considerations.

1) The basic tensor of the Einstein unified theory is a real tensor $g_{\lambda \mu}$. We denote by $h_{\lambda \mu}$ and $k_{\lambda \mu}$ its symmetric and skew-symmetric part respectively. As far as $h_{\lambda \mu}$ is concerned, we assume throughout this paper that its signature is $-\ldots+$ Hence its determinant is different from zero so that there exists an unique tensor $h^{\lambda \nu}=h^{\nu \lambda}$ satisfying the condition

$$
h_{\lambda \mu} h^{\lambda \nu}=\delta_{\mu}^{\nu}
$$

(where $\delta_{\mu}^{\nu}$ is the Kronecker delta). The tensor $h_{\lambda \mu}$ plays the role of a metric tensor. In particular we lower superscripts by means of $h_{\lambda \mu}$ and raise subscripts by means of $h^{\lambda \nu}$. Moreover, we denote by $\left\{\begin{array}{c}\nu \\ \lambda \mu\end{array}\right\}$ the Christoffel symbols belonging to $h_{\lambda \mu}$, by $\nabla_{\mu}$ the corresponding symbol of covariant derivative and by

$$
\begin{gathered}
H_{\omega \mu \lambda}{ }^{\nu} \equiv \partial_{\mu}\left\{\begin{array}{c}
\nu \\
\lambda \omega
\end{array}\right\}-\partial_{\omega}\left\{\begin{array}{c}
\nu \\
\lambda \mu
\end{array}\right\}+\left\{\begin{array}{c}
\nu \\
\alpha \mu
\end{array}\right\}\left\{\begin{array}{c}
\alpha \\
\lambda \omega
\end{array}\right\}-\left\{\begin{array}{c}
\nu \\
\alpha \omega
\end{array}\right\}\left\{\begin{array}{c}
\alpha \\
\lambda \mu
\end{array}\right\} \\
\left(\partial_{\mu} \equiv \frac{\partial}{\partial x^{\mu}}\right)
\end{gathered}
$$

its curvature tensor.

There exists always at least one set of four covariant vectors ${ }^{1} u_{\lambda}, \ldots,{ }^{4} u_{\lambda}$ such that

$$
h_{\lambda \mu}=-{ }^{1} u_{\lambda}{ }^{1} u_{\mu}-{ }^{2} u_{\lambda}{ }^{2} u_{\mu}-{ }^{3} u_{\lambda}{ }^{3} u_{\mu}+{ }^{4} u_{\lambda}{ }^{4} u_{\mu},
$$

while $k_{\lambda \mu}$ assumes one of the following mutually exclusive forms

$$
\begin{aligned}
& k_{\lambda \mu}=2 \alpha^{1} u_{[\lambda}{ }^{2} u_{\mu]}+2 \beta^{3} u_{[\lambda}{ }^{4} u_{\mu]} \\
& k_{\lambda \mu}=2 \delta^{2} u_{[\lambda}\left({ }^{3} u_{\mu]}+\epsilon^{4} u_{\mu]}\right),
\end{aligned}
$$$$
\epsilon= \pm 1
$$

where $\alpha, \beta, \delta$ are scalars. If $\alpha \beta \neq 0$ then we say that $k_{\lambda \mu}$ belongs to the first class. If one of the scalars $\alpha, \beta$ is equal to zero then $k_{\lambda \mu}$ will be referred to as belonging to the second class. Finally the tensor $k_{\lambda \mu}$ satisfying (1.3c) with $\delta \neq 0$ will be termed as belonging to the third class. The vectors ${ }^{1} u_{\lambda}, \cdots,{ }^{4} u_{\lambda}$ are uniquely defined by $g_{\lambda_{\mu}}$ (up to special Lorentz transformations) only in the case $(1.3 \mathrm{a}, \mathrm{b}$ ).

If we denote by $\mathfrak{g}, \mathfrak{h}, \mathfrak{f}$ the determinants of $g_{\lambda \mu}, h_{\lambda \mu}$ and $k_{\lambda \mu}$ respectively and put 


$$
g \equiv \frac{\mathfrak{g}}{\mathfrak{h}}, \quad k \equiv \frac{\mathfrak{t}}{\mathfrak{h}},
$$

we have

$$
g=1+\frac{1}{2} k_{\lambda \mu} k^{\lambda \mu}+k .
$$

According to our assumption about the signature of $h_{\lambda \mu}$ we have

$$
\mathfrak{h}<0 \text {. }
$$

On the other hand $\mathfrak{t} \geqq 0$, so that
(1.6) b)
$k \leqq 0$.

As far as $g$ is concerned we may have

$$
g \gtreqless 0 .
$$

Later on we shall see that the equality sign has to be excluded.

The classes of the field $k_{\lambda \mu}$ may be characterized algebraically by means of the scalar

$$
D\left(k_{\lambda \mu}\right)=\left(\frac{1}{2} k_{\alpha \beta} k^{\alpha \beta}\right)^{2}-4 k .
$$

The field $k_{\lambda \mu}$ belongs to the $\left\{\begin{array}{l}\text { first } \\ \text { second class } \\ \text { third }\end{array}\right.$

if and only if $\left\{\begin{array}{l}k D\left(k_{\lambda \mu}\right) \neq 0 \\ k=0, D\left(k_{\lambda \mu}\right) \neq 0 \\ k=0, D\left(k_{\lambda \mu}\right)=0 .\end{array}\right.$

2) Consider $g_{\lambda \mu}$ as auxiliary variables. Then a necessary and sufficient condition that the system

$$
\partial_{\omega} g_{\lambda \mu}=\Gamma_{\lambda \omega}^{\alpha} g_{\alpha \mu}+\Gamma_{\omega \mu}^{\alpha} g_{\lambda \alpha}
$$

admit one solution only is (cfr. [7]) either

$$
g \neq 0 \text { if } k \neq 0
$$

or

$$
g(g-2) \neq 0 \text { if } k=0 .
$$

In order to see clearly the significance of the sign of $\mathfrak{g}$ we choose a coordinate system in which the vectors ${ }^{a} u_{\lambda}$ involved in (1.3) have the components 
${ }^{a} u_{\lambda}=\delta_{\lambda}^{a}$ at a point $P$. Then the components of $g_{\lambda \mu}$ are given by one of the matrices

$$
\left(\left(\begin{array}{cccc}
-1 & \alpha & 0 & 0 \\
-\alpha & -1 & 0 & 0 \\
0 & 0 & -1 & \beta \\
0 & 0 & -\beta & 1
\end{array}\right)\right), \quad\left(\left(\begin{array}{cccc}
-1 & 0 & 0 & 0 \\
0 & -1 & \delta & \epsilon \delta \\
0 & -\delta & -1 & 0 \\
0 & -\epsilon \delta & 0 & 1
\end{array}\right)\right)
$$

Hence for the third class as well as for the second class with $\beta=0$ we have always $\mathfrak{g}<0, e . g ., g>0$. On the other hand the equation (1.5) holds even for the remaining case. Hence, if we allow only continuous change of the field $k_{\lambda \mu}$ we have to assume even in the remaining cases $g>0$. Consequently we shall deal in this paper with the cases only where besides $(1.8 \mathrm{a}, \mathrm{b})$ we have also
$(1.8) \mathrm{c})$
$\mathfrak{g}<0$
d)
$g>0$. $^{*}$

The unique solution $\Gamma_{\lambda \mu}^{\nu}$ of (1.7) has the form

$$
\Gamma_{\lambda \mu}^{\nu}=\left\{\begin{array}{c}
\nu \\
\lambda \mu
\end{array}\right\}+S_{\lambda \mu}{ }^{\nu}+U^{\nu}{ }_{\lambda \mu} .
$$

Here $S_{\lambda \mu}{ }^{\nu}=S_{[\lambda \mu]}{ }^{\nu}$ and $U^{\nu}{ }_{\lambda \mu}=U_{(\lambda \mu)}^{\nu}$ are tensors satisfying the following conditions:

a)

$$
S_{\lambda \mu}{ }^{\nu}=\Gamma_{[\lambda \mu]}^{\nu},
$$

$$
U_{\lambda \mu}^{\nu}=2 h^{\nu \alpha} S_{\alpha(\lambda}^{\beta} k_{\mu) \beta}
$$

c)

$$
U_{(\nu \lambda \mu)}=0 \text {. }
$$

The tensor $S_{\lambda \mu}{ }^{\nu}$ is the unique solution of the system

$$
2 S_{\omega \mu \nu}=K_{\omega \mu \nu}+4 S_{\nu \beta \gamma} k_{[\omega}^{\left[{ }^{\beta}\right.} k_{\mu]}{ }^{\gamma]}+4 k_{[\omega}{ }^{\beta} S_{\mu] \beta \gamma} k_{\nu}^{\gamma}
$$

equivalent to

$$
2 S_{\omega \mu \nu}=K_{\omega \mu \nu}-4 U_{\alpha \nu[\mu} k_{\omega]}^{\alpha} .
$$

The tensor $K_{\omega \mu \nu}$ involved in (1.11) is defined as follows:

$$
\begin{aligned}
K_{\omega \mu \nu} & \equiv \nabla_{\omega} k_{\nu \mu}+\nabla_{\mu} k_{\omega \nu}+\nabla_{\nu} k_{\omega \mu} \\
& =3 \nabla_{[\omega} k_{\nu \mu]}+2 \nabla_{\nu} k_{\omega \mu} .
\end{aligned}
$$

\footnotetext{
* Equations (1.3) are based on Theorem (5.2) in [5]. However, in this theorem we have $\beta<1$ (so that automatically $g>0$ ), for we confined ourselves in the corresponding proof to the case where for $\rho_{3} \neq 0$ we have $\rho_{3}>0, \rho_{4}>0$. For $\rho_{3}<0, \rho_{4}<0$ we obtain $\beta>1$ so that $g<0$.
} 
The tensor $U^{\nu}{ }_{\lambda \mu}$ may be obtained independently of $S_{\lambda \mu}{ }^{\nu}$ as the unique solution of the system*

(1.13) a) $\quad U_{\alpha \beta \gamma}\left[\delta_{\nu \lambda \mu}^{\alpha \beta \gamma}-2 k_{(\mu}^{\beta} k_{\lambda)}{ }^{\alpha} \delta_{\nu}^{\gamma}+2 k_{\nu}{ }^{\alpha} k_{(\mu}{ }^{\beta} \delta_{\lambda)}^{\gamma}\right]=-k_{(\mu}{ }^{\beta} K_{\lambda) \nu \beta}$.

Moreover,

$$
2 U^{\alpha \mu} \equiv 2 U_{\mu}=\partial_{\mu} \ln g .
$$

3) If we denote by $D_{\mu}$ the symbol of covariant derivative based on $\Gamma_{\lambda \mu}^{\nu}$, then (1.7) is equivalent to

$$
D_{\omega} g_{\lambda \mu}=2 S_{\omega \mu}^{\alpha} g_{\lambda \alpha}
$$

so that in particular

b) $\quad D_{\omega} h_{\lambda \mu}=2 S_{\omega(\mu}{ }^{\alpha} g_{\lambda) \alpha}=2 S_{\omega(\mu \lambda)}+U_{\omega \mu \lambda}$

c) $\quad D_{\omega} k_{\lambda \mu}=2 S_{\omega[\mu}^{\alpha} g_{\lambda] \alpha}=2 S_{\omega[\mu \lambda]}+2 S_{\omega[\mu}^{\alpha} k_{\lambda] \alpha}$.

Moreover, if we put

$$
S_{\lambda} \equiv S_{\lambda \alpha}{ }^{\alpha}
$$

we have

$$
D_{\lambda} \mathfrak{g}=2 S_{\lambda} \mathfrak{g} .
$$

2. Indicators $\mathfrak{F}^{\omega \mu \lambda \nu}$ and $\mathfrak{e}_{\omega \mu \lambda \nu}$. Denote by $\mathscr{F}^{\approx \mu \lambda \nu}$ a tensor density of weight -1 :

$$
\mathbb{E}^{\omega^{\prime} \mu^{\prime} \lambda^{\prime} \nu^{\prime}}=\frac{\partial x^{\omega^{\prime}}}{\partial x^{\alpha}} \frac{\partial x^{\mu^{\prime}}}{\partial x^{\beta}} \frac{\partial x^{\lambda^{\prime}}}{\partial x^{\gamma}} \frac{\partial x^{\nu^{\prime}}}{\partial x^{\delta}} \Delta^{-1} \mathbb{E}^{\alpha \beta \gamma \delta} \quad\left(\Delta=\operatorname{Det}\left|\frac{\partial x^{\alpha^{\prime}}}{\partial x^{\beta}}\right|\right)
$$

whose components are defined as follows: The components $\xi^{\omega \mu \lambda \nu}$ are skewsymmetric in all four indices. Moreover, $\overleftarrow{E}^{\omega \mu \lambda \nu}$ is equal to +1 or -1 according as the permutation

$$
\left(\begin{array}{llll}
1 & 2 & 3 & 4 \\
\omega & \mu & \lambda & \nu
\end{array}\right)
$$

is an even one or an odd one (and $\mathscr{E}^{\omega \mu \lambda \nu}=0$ in all remaining cases). This tensor density will be referred to as the contravariant indicator. The covariant indicator $\mathfrak{e}_{\omega \mu \lambda \nu}$ is a tensor density of weight +1 , skew-symmetric in all four subscripts, whose components are defined in the same way as the components of $\mathbb{E}^{\omega \mu \lambda \nu}$.

* Throughout this paper we use the abbreviation

$$
\delta_{\lambda_{1}}^{\nu_{1}^{1}}, \mathbf{u} \cdot \lambda_{s}^{\nu_{s}}=\delta_{\lambda_{1}}^{\nu_{1}} \cdots \delta_{\lambda_{s}}^{\nu_{s}^{s}}
$$$$
s=1,2, \cdots .
$$ 
In subsequent considerations we shall use some relationships between the two indicators. The first set of these relationships is dealt with in the following

Theorem (2.1). We have

a)

c)

$$
\begin{aligned}
& \mathscr{\digamma}^{\omega \mu \lambda \alpha} e_{\xi \eta \zeta \alpha}=6 \delta_{[\xi \eta \zeta]}^{\omega \mu \lambda}, \\
& \mathscr{F}^{\omega \mu \beta \alpha} e_{\xi \eta \beta \alpha}=4 \delta_{[\xi \eta]}^{\omega \mu}, \\
& \mathfrak{F}^{\omega \gamma \beta \alpha} e_{\xi \gamma \beta \alpha}=6 \delta_{\xi \xi}^{\omega} .
\end{aligned}
$$

Proof. First of all we have

$$
\delta_{[\xi \eta \zeta]}^{\omega \mu \lambda}=\frac{1}{6}\left[\delta_{\xi \eta \zeta}^{\omega \mu \lambda}+\delta_{\eta \zeta \xi}^{\omega \mu \lambda}+\delta_{\xi \xi \eta}^{\omega \mu \lambda}-\delta_{\eta \xi \zeta}^{\omega \mu \lambda}-\delta_{\xi \zeta \eta}^{\omega \mu \lambda}-\delta_{\zeta \eta \xi}^{\omega \mu \lambda}\right],
$$

so that obviously

$$
\mathfrak{F}^{\omega \mu \lambda \alpha} \mathfrak{e}_{\xi \eta \xi \alpha}=x \delta_{[\xi \eta \xi]}^{\omega \mu \lambda}
$$

where $x$ has to be found. Assuming $\omega \mu \lambda=\xi \eta \zeta=123$ one obtains at once $x=6$. Using (2.2a) one obtains by virtue of $(2.3)$

$$
\begin{aligned}
\mathbb{S}^{\xi \mu \beta \alpha} e_{\xi \eta \beta \alpha}^{\omega \mu \alpha} & =\delta_{\xi \eta \beta}^{\omega \mu \beta}+\delta_{\eta \beta \xi}^{\omega \mu \beta}+\delta_{\beta \xi \eta}^{\omega \mu \beta}-\delta_{\eta \xi \beta}^{\omega \mu \beta}-\delta_{\xi \beta \eta}^{\omega \mu \beta}-\delta_{\beta \eta \xi}^{\omega \mu \beta} \\
& =4 \delta_{\xi \eta}^{\omega \mu}+\delta_{\eta \xi}^{\omega \mu}+\delta_{\eta \xi}^{\omega \mu}-4 \delta_{\eta \xi}^{\omega \mu}-\delta_{\xi \eta}^{\omega \mu}-\delta_{\xi \eta}^{\omega \mu} \\
& =2\left(\delta_{\xi \eta}^{\omega \mu}-\delta_{\eta \xi}^{\omega \mu}\right)=4 \delta_{[\xi \eta]}^{\omega \mu} .
\end{aligned}
$$

Using now (2.2b) we obtain

$$
\left(^{\omega \gamma \beta \alpha} e_{\xi \gamma \beta \alpha}=2\left(\delta_{\xi \gamma}^{\omega \gamma}-\delta_{\gamma \xi}^{\omega \gamma}\right)=2\left(4 \delta_{\xi}^{\omega}-\delta_{\xi}^{\omega}\right)=6 \delta_{\xi}^{\omega} .\right.
$$

The second theorem deals with covariant components of the contravariant indicator as well as with the contravariant components of the covariant indicator.

Theorem (2.2). We have
a)
b)

$$
\begin{aligned}
\mathfrak{E}_{\omega \mu \lambda \nu} & =\mathfrak{e}_{\omega \mu \lambda \nu} \mathfrak{h} \\
\mathfrak{e}^{\omega \mu \lambda \nu} & =\mathfrak{E}^{\omega \mu \lambda \nu} \mathfrak{h}^{-1} .
\end{aligned}
$$

Proof. First we have

$$
\xi_{\omega \mu \lambda \nu}=\mathfrak{E}^{\alpha \beta \gamma \delta} h_{\alpha \omega} h_{\beta \mu} h_{\gamma \lambda} h_{\delta \nu}
$$

so that we must have

$$
\mathfrak{E}_{\omega \mu \lambda_{\nu}}=x \mathfrak{e}_{\omega \mu \lambda \nu}
$$

where $x$ is to be found. Comparing these two equations, we obtain at once $x=\mathfrak{h}$. The equation $(2.4 \mathrm{~b})$ may be provided in a similar manner. 
3. The field $F_{\lambda \mu}$. The first theorem in this section deals with the determinant

(3.1) $\quad \mathfrak{f}=\left|\begin{array}{cccc}0 & k_{12} & k_{13} & k_{14} \\ -k_{12} & 0 & k_{23} & k_{24} \\ -k_{13} & -k_{23} & 0 & k_{34} \\ -k_{14} & -k_{24} & -k_{34} & 0\end{array}\right|=\left(k_{12} k_{34}+k_{23} k_{14}+k_{31} k_{24}\right)^{2}$.

Theorem (3.1). The determinant $\mathfrak{t}$ satisfies the following relation

$$
64 \mathfrak{l}=\left(\mathfrak{E}^{\omega \mu \lambda \nu} k_{\omega \mu} k_{\lambda \nu}\right)^{2} .
$$

The proof follows at once from (3.1) and

$$
\check{E}^{\omega \mu \lambda \nu} k_{\omega \mu} k_{\lambda \nu}=8\left(k_{12} k_{34}+k_{23} k_{14}+k_{31} k_{24}\right) \text {. }
$$

The conditions (1.8) yield an unique tensor $* g^{\lambda \nu}$ satisfying the equation

$$
{ }^{*} g^{\nu \mu} g_{\lambda \mu}=\delta_{\lambda}^{\nu}
$$

We denote by ${ }^{*} g^{[\nu \mu]}$ its skew-symmetric part.

Theorem (3.2). The tensor ${ }^{*} g^{[\lambda \nu]}$ satisfies the following relation:

$$
*^{[\lambda \nu]}=\frac{1}{\mathfrak{g}}\left[\mathfrak{h} k^{\lambda \nu}+\kappa \frac{\sqrt{\mathfrak{l}}}{2} \mathfrak{E}^{\lambda \nu \alpha \beta} k_{\alpha \beta}\right]^{*} .
$$

Proof. First of all we have

$$
* g^{\nu \mu}=\frac{\partial \ln \mathfrak{g}}{\partial g_{\nu \mu}}
$$

and according to (1.5) and (3.2)

$$
\mathfrak{g}=\mathfrak{h}+\frac{\mathfrak{h}}{2} k_{\alpha \beta} k_{\gamma \delta} h^{\alpha \gamma} h^{\beta \delta}+\frac{1}{64}\left(\mathfrak{\mho}^{\alpha \beta \gamma \delta} k_{\alpha \beta} k_{\gamma \delta}\right)^{2} .
$$

Hence

$$
\begin{aligned}
*_{g^{[\nu \mu]}}^{[l]} & =\frac{\partial \ln \mathfrak{g}}{\partial k_{\nu \mu}}=\frac{1}{\mathfrak{g}}\left[\frac{\mathfrak{h}}{2} \frac{\partial}{\partial k_{\nu \mu}} k_{\alpha \beta} k_{\gamma \delta} h^{\alpha \gamma} h^{\beta \delta}+\frac{1}{64} \frac{\partial}{\partial k_{\nu \mu}}\left(\mathfrak{E}^{\alpha \beta \gamma \delta} k_{\alpha \beta} k_{\gamma \delta}\right)^{2}\right] \\
& =\frac{1}{\mathfrak{g}}\left[\mathfrak{h} k^{\nu \mu}+\frac{2}{32}\left(\mathfrak{F}^{\alpha \beta \gamma \delta} k_{\alpha \beta} k_{\gamma \delta}\right) \mathfrak{F}^{\nu \mu \gamma \delta} k_{\gamma \delta}\right] \\
& =\frac{1}{\mathfrak{g}}\left[\mathfrak{h} k^{\nu \mu}+\frac{\kappa}{2} \sqrt{\mathfrak{l} \mathfrak{E}^{\nu \mu \gamma \delta}} k_{\gamma \delta}\right] .
\end{aligned}
$$

$*_{\kappa}$ is the sign of $\mathscr{E}^{\omega \mu \lambda \nu} k_{\omega \mu} k_{\lambda \nu}$. If $\stackrel{(}{6 \mu \lambda \nu}^{\omega \mu} k_{\omega \mu} k_{\lambda \nu}=0$ then $\kappa$ is not defined but in this case we have $\mathfrak{f}=0$ so that the term containing $\kappa$ is not involved. 
In the next theorem we deal with the following tensors and tensor densities:

a)

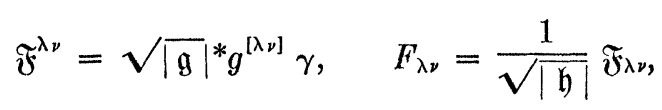

$$
(\gamma=\operatorname{sgn} \mathfrak{g}=-1)
$$

b)

$$
m_{\lambda \nu}=\frac{1}{2} \mathfrak{e}_{\lambda \nu \alpha \beta} \mathfrak{F}^{\alpha \beta}, \quad \mathfrak{m}^{\lambda \nu}=\sqrt{|\mathfrak{h}|} m^{\lambda \nu} .
$$

Theorem (3.3). We have
a)

$$
\mathfrak{F}^{\lambda \nu}=\frac{1}{\sqrt{|\mathfrak{g}|}}\left(\mathfrak{h} k^{\lambda \nu}+\kappa \frac{\sqrt{\mathfrak{f}}}{2} \mathfrak{E}^{\lambda \nu \alpha \beta} k_{\alpha \beta}\right)
$$
b)

$$
F_{\lambda \nu}=-\frac{1}{\sqrt{g}}\left(k_{\lambda \nu}+\kappa \frac{\sqrt{\mathfrak{f}}}{2} \mathrm{e}_{\lambda \nu \alpha \beta} k^{\alpha \beta}\right)
$$

and

a) $\quad m_{\lambda \nu}=\frac{1}{\sqrt{g}}\left[\kappa \sqrt{|k|} k_{\lambda \nu}-\frac{1}{2} \mathfrak{e}_{\lambda \nu \alpha \beta} \sqrt{|\mathfrak{h}|} k^{\alpha \beta}\right]$

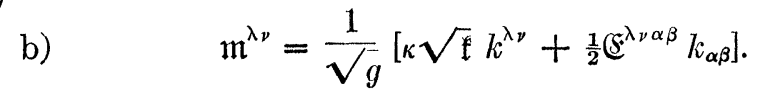

Moreover,

$$
\mathfrak{m}^{\lambda \nu}=-\frac{1}{2} \oiint^{\alpha \beta \lambda \nu} F_{\alpha \beta}, \quad \mathfrak{F}^{\lambda \nu}=\frac{1}{2} m_{\alpha \beta} \mathfrak{F}^{\alpha \beta \lambda \nu} .
$$

Proof. Equation (3.7a) follows at once from (3.4) and the first of (3.6a). Moreover, we obtain from (3.7a) and the second of (3.6a) by virtue of $(1.6 \mathrm{a}, \mathrm{b})$ and (2.4a)

$$
\begin{aligned}
F_{\lambda \nu} & =\frac{1}{\sqrt{|\mathfrak{g}|}}\left(-\sqrt{|\mathfrak{h}|} k_{\lambda \nu}+\frac{\kappa}{2} \sqrt{|k|} \mathfrak{e}_{\lambda \nu \alpha \beta} \mathfrak{h} k^{\alpha \beta}\right) \\
& =-\frac{1}{\sqrt{g}}\left(k_{\lambda \nu}+\kappa \frac{\sqrt{\bar{f}}}{2} \mathfrak{e}_{\lambda \nu \alpha \beta} k^{\alpha \beta}\right) .
\end{aligned}
$$

The first equation $(3.6 \mathrm{~b})$ yields by virtue of $(3.7 \mathrm{a}),(1.6 \mathrm{a}, \mathrm{b})$ and $(2.2 \mathrm{~b})$

$$
\begin{aligned}
m_{\lambda \nu} & =\frac{1}{2 \sqrt{|\mathfrak{g}|}}\left(\mathfrak{h} k^{\alpha \beta} \mathfrak{e}_{\lambda \nu \alpha \beta}+\kappa \frac{\sqrt{\mathfrak{f}}}{2} \mathfrak{e}_{\lambda \nu \alpha \beta} \mathscr{E}^{\alpha \beta \gamma \delta} k_{\gamma \delta}\right) \\
& =\frac{1}{2 \sqrt{g}}\left(-\sqrt{|\mathfrak{h}|} \mathfrak{e}_{\lambda \nu \alpha \beta} k^{\alpha \beta}+2 \kappa \sqrt{\frac{\mathfrak{f}}{|\mathfrak{h}|}} k_{\lambda \nu}\right) \\
& =\frac{1}{\sqrt{g}}\left[\kappa \sqrt{|k|} k_{\lambda \nu}-\frac{1}{2} \sqrt{|\mathfrak{h}|} \mathfrak{e}_{\lambda \nu \alpha \beta} k^{\alpha \beta}\right] .
\end{aligned}
$$


The second equation of (3.6b) together with (3.8a), (1.6a, b) and (2.4b) leads to

$$
\begin{aligned}
\mathrm{m}^{\lambda \nu} & =\frac{\sqrt{|\mathfrak{h}|}}{\sqrt{\bar{g}}}\left[\kappa \sqrt{|k|} k^{\lambda \nu}-\frac{1}{2} \mathfrak{E}^{\lambda \nu \alpha \beta} \sqrt{|\mathfrak{h}|} \mathfrak{h}^{-1} k_{\alpha \beta}\right] \\
& =\frac{1}{\sqrt{g}}\left[\kappa \sqrt{\mathfrak{f}} k^{\lambda \nu}+\frac{1}{2} \mathfrak{E}^{\lambda \nu \alpha \beta} k_{\alpha \beta}\right] .
\end{aligned}
$$

Moreover, by virtue of (3.6) and (2.4)

$$
\begin{aligned}
\mathrm{m}^{\lambda \nu}= & \sqrt{|\mathfrak{h}|} m^{\lambda \nu}=\frac{1}{2} \sqrt{|\mathfrak{h}|} \mathfrak{h}^{-1} \mathfrak{F}^{\lambda \nu \alpha \beta} \mathfrak{F}_{\alpha \beta}=-\frac{1}{2} \mathfrak{E}^{\lambda \nu \alpha \beta} F_{\alpha \beta} \\
& \frac{1}{2} m_{\alpha \beta} \mathfrak{E}^{\alpha \beta \lambda \nu}=\frac{1}{4} \mathfrak{e}_{\alpha \beta \gamma \delta} \mathfrak{E}^{\alpha \beta \lambda \nu} \mathfrak{F}^{\gamma \delta}=\mathfrak{F}^{\lambda \nu} .
\end{aligned}
$$

4. The field $m_{\lambda v}$. In this section we shall investigate the relationship between $k_{\lambda \mu}$ and $m_{\lambda \mu}$. In the first theorem we denote by $\mathfrak{m}$ the determinant of $m_{\lambda \mu}$ so that obviously

$$
64 \mathfrak{m}=\left(\Im^{\alpha \beta \gamma \delta} m_{\alpha \beta} m_{\gamma \delta}\right)^{2} .
$$

Theorem (4.1). The determinants $\mathfrak{m}$ and $\mathfrak{f}$ are related by

$$
\mathfrak{m}=\mathfrak{f}
$$

b) $\quad\left(\mathfrak{F}^{\omega \mu \lambda \nu} m_{\omega \mu} m_{\lambda \nu}=-8 \kappa \sqrt{\overline{\mathfrak{l}}}\right.$.

Proof. First of all we have

$$
\begin{aligned}
\mathfrak{E}^{\omega \mu \lambda \nu} m_{\omega \mu} m_{\lambda \nu}=\frac{1}{g} \mathfrak{F}^{\omega \mu \lambda \nu}\left[\kappa \sqrt{|k|} \mid k_{\omega \mu}-\frac{1}{2} \mathfrak{e}_{\omega \mu \alpha \beta} \sqrt{|\mathfrak{h}|} k^{\alpha \beta}\right] \\
\cdot\left[\kappa \sqrt{|k|} k_{\lambda \nu}-\frac{1}{2} \mathfrak{e}_{\lambda \nu \gamma \delta} \sqrt{|\mathfrak{h}|} k^{\gamma \delta}\right] .
\end{aligned}
$$

But

$$
\begin{aligned}
& \mathfrak{C}^{\omega \mu \lambda \nu}(\kappa \sqrt{|k|})^{2} k_{\omega \mu} k_{\lambda \nu}=8 \kappa \sqrt{\overline{\mathfrak{t}}}|k| \\
& -\frac{1}{2} \mathfrak{F}^{\omega \mu \lambda \nu} \mathfrak{e}_{\omega \mu \alpha \beta} \sqrt{|\mathfrak{h}|} k^{\alpha \beta} \kappa \sqrt{|k|} k_{\lambda \nu}=-2 \kappa \sqrt{\mathfrak{f}} k_{\alpha \beta} k^{\alpha \beta} \\
& =-\frac{1}{2} \mathfrak{E}^{\omega \mu \lambda \nu} e_{\lambda \nu \gamma \delta} \sqrt{|\mathfrak{h}|} k^{\gamma \delta} \kappa \sqrt{|k|} k_{\omega \mu} \\
& \frac{1}{4} \mathfrak{F}^{\omega \mu \lambda \nu} \mathfrak{e}_{\lambda \nu \gamma \delta} \mathfrak{e}_{\omega \mu \alpha \beta}(\sqrt{|\mathfrak{h}|})^{2} k^{\alpha \beta} k^{\gamma \delta}=\mathfrak{e}_{\gamma \delta \alpha \beta}|\mathfrak{h}| k^{\alpha \beta} k^{\gamma \delta} \\
& =-\S^{\gamma \delta \alpha \beta} k_{\alpha \beta} k_{\gamma \delta}=-8 \kappa \sqrt{\bar{t}} \text {. }
\end{aligned}
$$

Hence

$$
\left(^{\omega \mu \lambda \nu} m_{\omega \mu} m_{\lambda \nu}=-\frac{8 \kappa \sqrt{\overline{\mathfrak{f}}}}{g}\left(1+\frac{1}{2} k_{\alpha \beta} k^{\alpha \beta}+k\right)=-\frac{8 \kappa \sqrt{\mathfrak{f}}}{g} g=-8 \kappa \sqrt{\mathfrak{f}} .\right.
$$


Equation (4.2a) follows from (4.2b), (4.1) and (3.2). If $k=0$, then we have $\mathfrak{m}=0$ by virtue of $(4.2 \mathrm{a})$, and (4.2b) reduces to the identity $0=0$ because of (4.1).

Theorem (4.2). Equations (3.8a) admit the unique inverse solution

$$
\begin{aligned}
& k_{\lambda \nu}=\frac{\sqrt{g}}{1-m}\left[\frac{1}{2} \sqrt{|\mathfrak{h}|} \mathfrak{e}_{\lambda \nu \alpha \beta} m^{\alpha \beta}-\mu \sqrt{|m|} m_{\lambda \nu}\right]^{*} \\
&\left(m=\mathfrak{m} / \mathfrak{h}, \quad \mu=\operatorname{sgn}\left(\xi^{\omega \mu \lambda \nu} m_{\omega \mu} m_{\lambda \nu}\right) .\right.
\end{aligned}
$$

Proof. Let us put

$$
k_{\lambda \nu}=a e_{\lambda \nu \alpha \beta} m^{\alpha \beta}+b m_{\lambda v}
$$

where $a$ and $b$ are to be found. Substituting from (4.6) into (3.8a) we obtain $m_{\lambda \nu}=\frac{1}{\sqrt{g}}\left[\kappa \sqrt{|k|}\left(\mathfrak{a e}_{\lambda \nu \alpha \beta} m^{\alpha \beta}+b m_{\lambda \nu}\right)-\frac{1}{2} \mathfrak{e}_{\lambda \nu \alpha \beta} \sqrt{|\mathfrak{h}|}\left(\mathfrak{a}\left(\mathfrak{E}^{\gamma \delta \alpha \beta} m_{\gamma \delta} \mathfrak{h}^{-1}+b m^{\alpha \beta}\right)\right]\right.$.

But

$$
\begin{gathered}
-\frac{1}{2} \mathfrak{e}_{\lambda \nu \alpha \beta} \sqrt{|\mathfrak{h}|} \mathfrak{a} \mathfrak{E}^{\gamma \delta \alpha \beta} m_{\gamma \delta} \mathfrak{h}^{-1}=\frac{2 \mathfrak{a}}{\sqrt{|\mathfrak{h}|}} m_{\lambda \nu} \\
-\frac{1}{2} \mathfrak{e}_{\lambda \nu \alpha \beta} \sqrt{|\mathfrak{h}|} b m^{\alpha \beta}+\kappa \sqrt{|k|} \mathfrak{a} \mathfrak{e}_{\lambda \nu \alpha \beta} m^{\alpha \beta}=\mathfrak{e}_{\lambda \nu \alpha \beta} m^{\alpha \beta}\left(\kappa \sqrt{\left.|k| \mathfrak{a}-\frac{b}{2} \sqrt{|\mathfrak{h}|}\right)}\right.
\end{gathered}
$$

so that

$$
m_{\lambda \nu}=\frac{1}{\sqrt{g}}\left[\left(\kappa \sqrt{|k|} b+\frac{2 \mathfrak{a}}{\sqrt{|\mathfrak{h}|}}\right) m_{\lambda \nu}+\mathfrak{e}_{\lambda \nu \alpha \beta} m^{\alpha \beta}\left(\kappa \sqrt{|k| \mathfrak{a}}-\frac{b}{2} \sqrt{|\mathfrak{h}|}\right)\right] .
$$

This equation becomes an identity for any $m_{\lambda_{\nu}}$ if and only if

$$
\begin{aligned}
b & =\frac{\sqrt{g}}{1+|k|} \kappa \sqrt{|k|}=\frac{-\sqrt{g}}{1-m} \mu \sqrt{|m|} \\
\mathfrak{a} & =\frac{\sqrt{|\mathfrak{h}|}}{2} \frac{\sqrt{g}}{1+|k|}=\frac{\sqrt{|\mathfrak{h}|}}{2} \frac{\sqrt{g}}{1-m} .
\end{aligned}
$$

Substituting (4.7) into (4.6) we obtain $(4.5)$.

In the next theorem we introduce a scalar $M$ defined in terms of the tensor $h_{\lambda \mu}+m_{\lambda \mu}$ in the same way that $g$ was defined in terms of $g_{\lambda \mu}=h_{\lambda \mu}+k_{\lambda \mu}$ (cfr. (1.5)):

$$
M \equiv 1+\frac{1}{2} m_{\alpha \beta} m^{\alpha \beta}+m
$$

* Since $m \leqq 0$ we have always $1-m \neq 0$. 
Theorem (4.3). If

$$
(m+1) M \neq 0^{*}
$$

then the tensor $m_{\lambda_{\mu}}$ uniquely defines the tensor $k_{\lambda \mu}$ by means of (4.5), where

$$
g=\frac{2(1+m)(1-m)^{2}}{2(1-m)^{2}+m_{\alpha \beta} m^{\alpha \beta}(1+m)+8 m}=\frac{(1-m)^{2}}{M}
$$

Proof. First we obtain from (4.5)

$$
k_{\alpha \beta} k^{\alpha \beta}=-\frac{g}{(1-m)^{2}}\left[m^{\alpha \beta} m_{\alpha \beta}(1+m)+8 m\right] .
$$

Substituting from (4.9) and (4.2) into (1.5) we obtain

$$
g\left\{1+\frac{1}{2(1-m)^{2}}\left[m^{\alpha \beta} m_{\alpha \beta}(1+m)+8 m\right]\right\}=1+m .
$$

If $1+m=0$, then the factor of $g$ in (4.10) is equal to zero. If however, $(1+m) M \neq 0$, we obtain (4.9) from (4.10).

Theorem (4.4). The fields $m_{\lambda \mu}$ and $k_{\lambda \mu}$ belong to the same class and their canonical form is expressed in terms of the same set of vectors ${ }^{a} u_{\lambda}$.

Proof. The class of $m_{\lambda \mu}$ is characterized by $m$ and

$$
D\left(m_{\lambda \mu}\right) \equiv\left(\frac{1}{2} m_{\alpha \beta} m^{\alpha \beta}\right)^{2}-4 m .
$$

Assume first $k \neq 0$. Then we must have $D\left(k_{\lambda \mu}\right) \neq 0 \dagger$ and $k_{\lambda \mu}$ belongs to the first class. According to (4.2) we have also $\mathfrak{m} \neq 0$ and by the same token $D\left(m_{\lambda \mu}\right) \neq 0$, so that $m_{\lambda \mu}$ belongs to the first class. Assume now that $k_{\lambda \mu}$ belongs to the second class so that $k=0, D\left(k_{\lambda \mu}\right) \neq 0$. From (4.2) we have $\mathfrak{m}=0$, and (4.9) yields

$$
k_{\alpha \beta} k^{\alpha \beta}=-g m^{\alpha \beta} m_{\alpha \beta}
$$

so that $D\left(m_{\lambda \mu}\right) \neq 0$ (as a consequence of $D\left(k_{\lambda \mu}\right) \neq 0$ ). Hence $m_{\lambda \mu}$ belongs to the second class. A similar method shows that if $k_{\lambda \mu}$ belongs to the third class the same holds for the tensor $m_{\lambda \mu}$. The obvious identities

$$
\begin{aligned}
& \mathfrak{e}_{\lambda \nu \omega \mu}{ }^{1} u^{\lambda{ }^{2}} u^{\nu 1} u^{\omega 2} u^{\mu}=0, \\
& \mathfrak{e}_{\lambda \nu \omega \mu} u^{\lambda} u^{4} u^{\nu 3} u^{\omega 4} u^{\mu}=0
\end{aligned}
$$

* The case $m+1=0$ will be dealt with in Theorem (4.5).

$\dagger C f r$. Theorem (4.2) in [5]. 
show that the bivectors

$$
\begin{aligned}
& { }^{1} u^{\left[\lambda{ }^{2}\right.} u^{\nu]} \quad \text { and } \quad^{\lambda \nu \omega \mu 1} u_{\omega}{ }^{2} u_{\mu} \text {, } \\
& { }^{3} u^{\left[\lambda{ }^{4}\right.} u^{\nu]} \quad \text { and } \quad\left(^{\lambda \nu \omega \mu}{ }^{3} u_{\omega}{ }^{4} u_{\mu}\right.
\end{aligned}
$$

are totally perpendicular so that $\left(^{\lambda \nu \omega \mu 1} u_{\omega}{ }^{2} u_{\mu}\left(\aleph^{\lambda \nu \omega \mu}{ }^{3} u_{\omega}{ }^{4} u_{\mu}\right)\right.$ is proportional to ${ }^{3} u^{\left[\lambda{ }^{4}\right.} u^{\mu]}\left({ }^{1} u^{[\lambda 2} u^{\mu]}\right)$. This fact together with (3.8a) yields at once the proof of the remaining statement in our theorem.

We are now able to complete Theorem (4.3).

Theorem (4.5). If

$$
m+1=0
$$

we have also

$$
k+1=0 \text {. }
$$

If

$$
M \neq 0
$$

then the equation (4.11b) together with (4.5) defines two tensors $k_{\lambda \nu}$ (up to sign) by means of $m_{\lambda \mu}$.

Proof. It is sufficient to prove this statement at a point $P$ referred to a special coordinate system. According to $(4.11 \mathrm{~b}$ ) (which is an immediate consequence of (4.11a) and (4.2)) the field $k_{\lambda \mu}$ (as well as $m_{\lambda \mu}$ ) belongs to the first class. Hence if we use the coordinate system in which $g_{\lambda \mu}$ is given by the first of the two matrices preceding $(1.8 \mathrm{c}, \mathrm{d})$ we have from $(4.11 \mathrm{~b})$

$$
\begin{aligned}
& \text { a) } \beta=\epsilon \alpha^{-1}, \quad \text { b) } \kappa=\epsilon, \quad \text { c) } g=\alpha^{2}-\frac{1}{\alpha^{2}}=\frac{\alpha^{4}-1}{\alpha^{2}}>0 * \\
& \quad(\epsilon= \pm 1),
\end{aligned}
$$

and similarly (by virtue of the last statement of Theorem (4.4))

$$
m_{34}=\epsilon^{\prime} m_{12}^{-1}, \quad \text { b) } \mu=\epsilon^{\prime} .
$$

Consequently equation (4.5) yields for $\lambda \nu=12$

$$
\alpha=-\frac{\sqrt{\alpha^{4}-1}}{2|\alpha|} \epsilon^{\prime} \frac{1+m_{12}^{2}}{m_{12}}
$$

or

$$
4 \alpha^{4}=\left(\alpha^{4}-1\right)\left(\frac{1+m_{12}^{2}}{m_{12}}\right)^{2}
$$

* According to $(1.8 \mathrm{c}, \mathrm{d})$ we must have $\alpha^{2}>1$. 
We are dealing only with real tensors $k_{\lambda \mu}$, so that the last equation yields

$$
\alpha= \pm \sqrt{\frac{1+m_{12}^{2}}{\left|1-m_{12}^{2}\right|}}
$$

and this equation is not meaningless, for according to (4.11c) we must have $\left(m_{12}\right)^{2} \neq 1$. Combining this equation with (4.12a) we obtain two tensors $k_{\lambda \mu}$ (up to sign) in our special coordinate system at $P$. This result is obviously independent of the choice of coordinate system.

5. Continuation. In this section we are going to investigate the relationship between the fields $k_{\lambda \mu}, m_{\lambda \mu}$ and $F_{\lambda \mu}$.

Theorem (5.1). The following differential relations hold

a) $\quad 3 \nabla_{[\omega} m_{\mu \lambda]}=3 \partial_{[\omega} m_{\mu \lambda]}=\mathfrak{e}_{\omega \mu \lambda \nu} \nabla_{\alpha} \mathfrak{F}^{\alpha \nu}=\mathfrak{e}_{\omega \mu \lambda \nu} \partial_{\alpha} \mathfrak{F}^{\alpha \nu}$

b) $\quad 3 \nabla_{[\omega} F_{\mu \lambda]}=3 \partial_{[\omega} F_{\mu \lambda]}=-\mathfrak{e}_{\omega \mu \lambda \nu} \nabla_{\alpha} \mathfrak{m}^{\alpha \nu}=-\mathfrak{e}_{\omega \mu \lambda \nu} \partial_{\alpha} \mathfrak{m}^{\alpha \nu}$.

Proof. The second equation (3.9) yields

$$
\nabla_{\alpha} \mathfrak{F}^{\alpha \nu}=\frac{1}{2} \mathscr{E}^{\alpha \nu \gamma \delta} \nabla_{\alpha} m_{\gamma \delta}=\frac{1}{2} \overleftarrow{\digamma}^{\alpha \gamma \delta \nu} \nabla_{\alpha} m_{\gamma \delta}
$$

so that

$$
\mathrm{e}_{\omega \mu \lambda \nu} \nabla_{\alpha} \mathfrak{F}^{\alpha \nu}=\frac{1}{2} \mathrm{e}_{\omega \mu \lambda \nu} \mathfrak{F}^{\alpha \gamma \delta \nu} \nabla_{\alpha} m_{\gamma \delta}=3 \nabla_{[\omega} m_{\mu \lambda]} .
$$

On the other hand the first equation (3.9) yields

$$
\nabla_{\alpha} \mathfrak{m}^{\alpha \nu}=-\frac{1}{2} \xi^{\alpha \nu \gamma \delta} \nabla_{\alpha} F_{\gamma \delta}
$$

so that

$$
-e_{\omega \mu \lambda \nu} \nabla_{\alpha} \mathfrak{m}^{\alpha \nu}=3 \nabla_{[\omega} F_{\mu \lambda]}
$$

On the other hand

$$
\nabla_{\lambda} \mathfrak{m}^{\lambda \nu}=\partial_{\lambda} \mathfrak{m}^{\lambda \nu}+\left\{\begin{array}{c}
\lambda \\
\alpha \lambda
\end{array}\right\} \mathfrak{m}^{\alpha^{\alpha \nu}}+\left\{\begin{array}{c}
\nu \\
\alpha \lambda
\end{array}\right\} \mathfrak{m}^{\lambda \alpha}-\left\{\begin{array}{c}
\alpha \\
\alpha \lambda
\end{array}\right\} \mathfrak{m}^{\lambda_{\nu}}=\partial_{\lambda} \mathfrak{m}^{\lambda \nu}
$$

and similarly

$$
\nabla_{\lambda} \mathfrak{F}^{\lambda \nu}=\partial_{\lambda} \mathfrak{F}^{\lambda \nu}
$$

Moreover,

$$
\nabla_{[\omega} m_{\mu \lambda]}=\partial_{[\omega} m_{\mu \lambda]}+\left\{\begin{array}{c}
\alpha \\
{[\mu \omega]}
\end{array}\right\} m_{\lambda] \alpha}-\left\{\begin{array}{c}
\alpha \\
{[\lambda \omega}
\end{array}\right\} m_{\mu] \alpha}=\partial_{[\omega} m_{\mu \lambda]} .
$$


Theorem (5.2). The requirement

$$
\partial_{\lambda} \mathfrak{F}^{\lambda \nu}=0
$$

equivalent to

$$
\nabla_{\lambda} \mathfrak{F}^{\lambda \nu}=0
$$

is equivalent also to

a) $\kappa \sqrt{|k|} \nabla_{[\mu} k_{\lambda \nu]}=\sqrt{g} U_{[\mu} m_{\lambda \nu]}-\kappa \sqrt{|k|} P_{[\mu} k_{\lambda \nu]}+\frac{\sqrt{|\mathfrak{h}|}}{2} \mathfrak{e}_{\alpha \beta[\mu \lambda} \nabla_{\nu]} k^{\alpha \beta}$,

(5.3) b) $\kappa \sqrt{|k|} \mathfrak{F}^{\omega \mu \lambda \nu} \nabla_{\mu} k_{\lambda \nu}=\Im^{\omega \mu \lambda \nu}\left(\sqrt{g} U_{\mu} m_{\lambda \nu}-\kappa \sqrt{|k|} P_{\mu} k_{\lambda \nu}\right)$

$$
+2 \sqrt{|\mathfrak{h}|} \nabla_{\alpha} k^{\omega \alpha}
$$

where

$$
2 P_{\mu} \equiv \partial_{\mu} \ln k=\partial_{\mu} \ln m
$$

Proof. The second equation (5.4) as well as the equivalance of (5.2a) and $(5.2 \mathrm{~b})$ is obvious. The equation (1.13b), (5.1a), (3.8a) and (5.2) yield at once (5.3a). Upon multiplying this latter equation by $\stackrel{5}{\omega \mu \lambda \nu}^{\omega a n e}$ obtains $(5.3 \mathrm{~b})$.

6. Curvature tensor. In this section we shall investigate the relationships among the following tensors: the curvature tensor $R_{\omega \mu \lambda}{ }^{\nu}$ of the connection $\Gamma_{\lambda \mu}^{\nu}$

$$
R_{\omega \mu \lambda}{ }^{\nu}=\partial_{\mu} \Gamma_{\lambda \omega}^{\nu}-\partial_{\omega} \Gamma_{\lambda \mu}^{\nu}+\Gamma_{\alpha \mu}^{\nu} \Gamma_{\lambda \omega}^{\alpha}-\Gamma_{\alpha \omega}^{\nu} \Gamma_{\lambda \mu}^{\alpha} ;
$$

the contracted curvature tensors

b)

$$
R_{\mu \lambda}=R_{\alpha \mu \lambda}^{\alpha}
$$

c)

$$
V_{\omega \mu}=R_{\omega \mu \alpha}^{\alpha}
$$

the Einstein tensor $P_{\lambda \mu}$,

(6.1) d) $P_{\lambda \mu}=\partial_{\alpha} \Gamma_{\lambda \mu}^{\alpha}-\Gamma_{\lambda \beta}^{\alpha} \Gamma_{\alpha \mu}^{\beta}-\frac{1}{2}\left(\partial_{\mu} \Gamma_{(\lambda \alpha)}^{\alpha}+\partial_{\lambda} \Gamma_{(\mu \alpha)}^{\alpha}\right)+\Gamma_{\lambda \mu}^{\alpha} \Gamma_{(\alpha \beta)}^{\beta}$;

the tensor of torsion

$$
S_{\lambda \mu}^{\nu}=\Gamma_{[\lambda \mu]}^{\nu}
$$

and the contracted tensor of torsion

$$
S_{\lambda} \equiv S_{\lambda \alpha}{ }^{\alpha} .
$$


The curvature tensor satisfies the following identities

a)

$$
2 D_{[\omega} D_{\mu]} w_{\lambda} \equiv R_{\omega \mu \lambda}{ }^{\nu} w_{\nu}+2 S_{\omega \mu \mu}^{\alpha} D_{\alpha} w_{\lambda}
$$

b)

$$
2 D_{[\omega} D_{\mu]} v^{\nu} \equiv-R_{\omega \mu \lambda}{ }^{\nu} v^{\nu}+2 S_{\omega \mu}{ }^{\alpha} D_{\alpha} v^{\nu}
$$

for any vector fields $w_{\lambda}, v^{v}$ whatsoever. Other (known)* necessary identities will be proved in the following

Theorem (6.1). We have

a)

$$
R_{[\xi \omega \mu]}^{\nu}=+\left(4 S_{[\xi \omega}{ }^{\alpha} S_{\mu] \alpha}{ }^{\nu}+2 D_{[\xi} S_{\omega \mu]}{ }^{\nu}\right)
$$

b)

$$
D_{[\xi} R_{\omega \mu] \lambda}{ }^{\nu}=-2 S_{[\omega \mu}{ }^{\alpha} R_{\xi] \alpha \lambda}{ }^{\nu} .
$$

Proof. First of all we obtain from (6.2)

$$
\begin{aligned}
2 D_{[\xi}\left(D_{\omega} D_{\mu]} w_{\lambda}\right)=\left(D_{[\xi} R_{\omega \mu] \lambda}{ }^{\nu}\right) w_{\nu}+ & R_{[\omega \mu|\lambda|}{ }^{\nu} D_{\xi]} w_{\nu} \\
& +2\left(D_{[\xi} S_{\omega \mu]}^{\alpha}\right) D_{\alpha} w_{\lambda}+2 S_{[\omega \mu}^{\alpha} D_{\xi]} D_{\alpha} w_{\lambda}
\end{aligned}
$$

and

$$
2 D_{[\xi} D_{\omega}\left(D_{\mu]} w_{\lambda}\right)=R_{[\xi \mu \mu]}^{\alpha} D_{\alpha} w_{\lambda}+2 S_{[\xi \omega}{ }^{\alpha} D_{|\alpha|} D_{\mu]} w_{\lambda}
$$

$$
+R_{[\xi \omega|\lambda|}^{\alpha} D_{\mu]} w_{\alpha}
$$

Moreover, by virtue of (6.2)

$$
2 S_{[\xi \omega}^{\alpha} D_{|\alpha|} D_{\mu]} w_{\lambda}=2 S_{[\xi \omega}{ }^{\alpha}\left(D_{\mu]} D_{\alpha} w_{\lambda}+R_{|\alpha| \mu] \lambda}^{\beta} w_{\beta}+2 S_{|\alpha| \mu]}^{\beta} D_{\beta} w_{\lambda}\right) .
$$

Comparing these three equations we obtain

$$
\begin{aligned}
w_{\nu}\left[D_{[\xi} R_{\omega \mu] \lambda}{ }^{\nu}+2 S_{[\xi \omega}{ }^{\alpha} R_{\mu] \alpha \lambda}{ }^{\nu}\right] & +\left(D_{\alpha} w_{\nu}\right)\left[\delta_{[\xi}^{\alpha} R_{\omega \mu] \lambda}{ }^{\nu}+2 \delta_{\lambda}^{\nu} D_{[\xi} S_{\omega \mu]}^{\alpha}\right. \\
& \left.+4 S_{[\xi \omega}{ }^{\beta} S_{\mu] \beta}{ }^{\alpha} \delta_{\lambda}^{\nu}-R_{[\xi \omega \mu]}^{\alpha} \delta_{\lambda}^{\nu}-\delta_{[\mu}^{\alpha} R_{\xi \omega] \lambda}{ }^{\nu}\right]=0 .
\end{aligned}
$$

This equation is an identity for any vector $w_{\lambda}$ so that (6.3) must hold.

Corollary (6.1). We have

a) $\quad 2 R_{[\omega \mu]}+V_{\omega \mu}=2\left[D_{\alpha} S_{\omega \mu}{ }^{\alpha}+2 D_{[\omega} S_{\mu]}-2 S_{\omega \mu}{ }^{\alpha} S_{\alpha}\right]$,

$$
\begin{aligned}
\text { b) } \quad V_{\omega \mu} & =2\left(D_{[\omega} S_{\mu]}-S_{\omega \mu}{ }^{\alpha} S_{\alpha}\right)=2 \partial_{[\omega} S_{\mu]}, \\
\text { c) } & R_{[\omega \mu]}=\left(D_{\alpha} S_{\omega \mu}{ }^{\alpha}+\partial_{[\omega} S_{\mu]}\right)
\end{aligned}
$$

* (6.3b) is known as the "generalized" Bianchi identity. 
Proof. Summing for $\xi=\nu$ in (6.3a) we obtain (6.4a). In order to prove (6.4b) we first use equation

$$
V_{\omega \mu}=\partial_{\mu} \Gamma_{\alpha \omega}^{\alpha}-\partial_{\omega} \Gamma_{\alpha \mu}^{\alpha}
$$

following from (6.1a, c). Moreover, we have from (1.9) and (1.13b)

$$
\begin{aligned}
\Gamma_{\alpha \omega}^{\alpha} & =\left\{\begin{array}{c}
\alpha \\
\alpha \omega
\end{array}\right\}+S_{\alpha \omega}{ }^{\alpha}+U^{\alpha}{ }_{\alpha \omega} \\
& =\frac{1}{2} \partial_{\omega} \ln \mathfrak{h}+\frac{1}{2} \partial_{\omega} \ln \mathfrak{g}-\frac{1}{2} \partial_{\omega} \ln \mathfrak{h}-S_{\omega} \\
& =\frac{1}{2} \partial_{\omega} \ln \mathfrak{g}-S_{\omega} .
\end{aligned}
$$

Comparing $(6.5 \mathrm{a}, \mathrm{b})$ we obtain

$$
V_{\omega \mu}=-2 \partial_{[\mu} S_{\omega]}=-2\left[D_{[\mu} S_{\omega]}+S_{\omega \mu}^{\alpha} S_{\alpha}\right]
$$

and this equation is equivalent to (6.4b). Equation (6.4c) is an immediate consequence of $(6.4 \mathrm{a}, \mathrm{b})$.

Theorem (6.2). The tensors $R_{\lambda \mu}$ and $P_{\mu \lambda}$ are related by

$$
P_{\lambda \mu}+R_{\mu \lambda}=D_{\mu} S_{\lambda}
$$

Proof. Besides (6.5b) we have also

$$
\Gamma_{\omega \alpha}^{\alpha}=\frac{1}{2} \partial_{\omega} \ln \mathfrak{g}+S_{\omega},
$$

so that

$$
\Gamma_{(\lambda \alpha)}^{\alpha}=\frac{1}{2} \partial_{\lambda} \ln \mathfrak{g} .
$$

Now using $(6.1 \mathrm{a}, \mathrm{b})$ as well as (6.1d) one obtains by virtue of (6.7) and (6.8)

$$
\begin{aligned}
R_{\mu \lambda}+P_{\lambda \mu} & =\frac{1}{2} \partial_{\mu} \partial_{\lambda} \ln \mathfrak{g}+\partial_{\mu} S_{\lambda}+S_{\beta \alpha}^{\beta} \Gamma_{\lambda \mu}^{\alpha}-\frac{1}{2} \partial_{\mu} \partial_{\lambda} \ln \mathfrak{g} \\
& =\partial_{\mu} S_{\lambda}-\Gamma_{\lambda \mu}^{\alpha} S_{\alpha}=D_{\mu} S_{\lambda} .
\end{aligned}
$$

Theorem (6.3). Any two of the three following conditions:
(6.9) a)
$\check{\leftarrow}^{\omega \mu \lambda[\nu} R_{\omega \mu \lambda}^{\rho]}=0$,
b) $\quad R_{[\mu \lambda]}=0$,
c) $S_{\lambda}=\partial_{\lambda} S$

imply the third.

Proof. Let us multiply (6.9a) by $\mathfrak{e}_{\rho \eta \zeta^{\prime} \nu}$ :

$$
\begin{aligned}
2 \mathfrak{e}_{\rho \eta \xi \nu}\left(\xi^{\omega \mu \lambda[\nu} R_{\omega \mu \lambda}^{\rho]}\right. & =6 R_{[\rho \eta \xi]}^{\rho}+6 R_{[\eta \xi \nu]}^{\nu}=12 R_{[\alpha \eta \xi]}^{\alpha} \\
& =4\left(2 R_{[\eta \xi]}+V_{\eta \xi}\right) .
\end{aligned}
$$

This equation together with $(6.4 \mathrm{a}, \mathrm{b})$ lead at once to the proof of our statement. Note. A similar set of conditions (with $S=0$ ) has been used in [4]. 
Theorem (6.4). The condition

$$
D_{\nu} R_{[\omega \mu \lambda]}^{\nu}=4 R_{\alpha[\omega \lambda}{ }^{\beta} S_{\mu] \beta}{ }^{\alpha}+2 S_{[\omega \lambda}{ }^{\alpha} R_{\mu] \alpha}
$$

is equivalent to

$$
\partial_{[\omega} R_{\mu \lambda]}=0
$$$$
\left(=\nabla_{[\omega} R_{\mu \lambda]}\right) .
$$

Proof. First of all we have

$$
D_{[\omega} R_{\mu \lambda]}=\nabla_{[\omega} R_{\mu \lambda]}-R_{\alpha[\lambda} S_{\mu \omega]}^{\alpha}-S_{[\lambda \omega}{ }^{\alpha} R_{\mu] \alpha} .
$$

Moreover, we obtain from the Bianchi identity (6.3b) for $\xi=\nu$

(6.11) b) $D_{\nu} R_{\omega \mu \lambda}{ }^{\nu}+2 D_{[\mu} R_{\omega] \lambda}=-2\left[R_{\alpha \lambda} S_{\omega \mu}{ }^{\alpha}-R_{\alpha \omega \lambda}{ }^{\beta} S_{\mu \beta}{ }^{\alpha}+R_{\alpha \mu \lambda}{ }^{\beta} S_{\omega \beta}{ }^{\alpha}\right]$.

Hence,

$$
D_{\nu} R_{[\omega \mu \lambda]}^{\nu}-2 D_{[\omega} R_{\mu \lambda]}=-2\left[R_{\alpha[\lambda} S_{\omega \mu]}^{\alpha}+2 R_{\alpha[\mu \lambda}{ }^{\beta} S_{\omega] \beta}^{\alpha}\right] .
$$

The proof follows easily from $(6.11 \mathrm{a}, \mathrm{c})$.

7. Continuation. In this section we shall investigate the relationship of the tensors (6.1), (1.2) and

$$
H_{\mu \lambda} \equiv H_{\alpha \mu \lambda}{ }^{\alpha} .
$$

Theorem (7.1a). Equation (6.4c) is equivalent to

(7.2) a) $\quad R_{[\omega \mu]}=\nabla_{\alpha} S_{\omega \mu}{ }^{\alpha}+2 U^{\beta}{ }_{\alpha[\omega} S_{\mu] \beta}{ }^{\alpha}+\left(U_{\beta}+S_{\beta}\right) S_{\omega \mu}{ }^{\beta}+\partial_{[\omega} S_{\mu]}$.

The proof follows easily from $(6.4 \mathrm{c})$ and

$$
D_{\alpha} S_{\omega \mu}{ }^{\alpha}=\nabla_{\alpha} S_{\omega \mu}^{\alpha}+2 U_{\alpha[\omega}^{\beta} S_{\mu] \beta}{ }^{\alpha}+\left(U_{\beta}+S_{\beta}\right) S_{\omega \mu}{ }^{\beta} .
$$

In the next theorem we shall use the following abbreviation:

$$
\begin{aligned}
A_{\omega \mu} \equiv 2 \nabla^{\alpha} U_{\beta \alpha[\omega} k_{\mu]}{ }^{\beta} & +2 U^{\beta}{ }_{\alpha[\omega}\left(\frac{1}{2} K_{\mu] \beta}{ }^{\alpha}-k_{\mu]}{ }^{\gamma} U_{\gamma \beta}^{\alpha}\right. \\
& \left.+U^{\gamma \alpha}{ }_{\mu]} k_{\beta \gamma}\right)+U^{\beta}\left(\frac{1}{2} K_{\omega \mu \beta}-2 U_{\alpha \beta[\mu} k_{\omega]}{ }^{\alpha}\right)=-A_{\mu \omega .} .
\end{aligned}
$$

Theorem (7.1b). The equation (7.2) is equivalent to

$$
\text { b) } R_{[\omega \mu]}=\frac{1}{2} \nabla^{\alpha} K_{\omega \mu \alpha}+A_{\omega \mu}+\partial_{[\omega} S_{\mu]}+S^{\beta}\left(\frac{1}{2} K_{\omega \mu \beta}-2 U_{\alpha \beta[\mu} k_{\omega]}{ }^{\alpha}\right) \text {. }
$$

Proof. Substituting from (1.11b) into (7.2a) one obtains (7.2b).

Note. The tensor $U^{\nu}{ }_{\lambda \mu}$ may be computed independently from (1.13a), without knowledge of $S_{\lambda \mu}{ }^{\nu}$. Hence, the same is true for $A_{\omega \mu}$ and consequently only the last two terms on the right hand side of (7.2b) may be looked upon as dependent on $S_{\lambda \mu}{ }^{\nu}$. Moreover, we may prove the following Lemma important for later purposes. 
Lemma (7.1). The tensor $A_{\omega \mu}$ involved in (7.2b) does not contain the term $\nabla^{\alpha} K_{\omega \mu \alpha}$. Proof. The only tensor which could give rise to such terms in $A_{\omega \mu}$ is (according to $(1.10 \mathrm{~b}))$

$$
2 \nabla^{\alpha} U_{\beta \alpha[\omega} k_{\mu]}^{\beta}=2 \nabla^{\alpha}\left(S_{\beta \alpha \gamma} k_{[\omega}^{\gamma}+k_{\alpha \gamma} S_{\beta[\omega}{ }^{\gamma}\right) k_{\mu]}{ }^{\beta} .
$$

Substituting in the right hand term of this equation from (1.11a) we see that the first term on the right hand side gives rise to

$$
\left(\nabla^{\alpha} K_{\beta \alpha \gamma}\right) k_{[\omega}^{\gamma} k_{\mu]}^{\beta},
$$

and no term gives rise to $\nabla^{\alpha} K_{\omega \mu \alpha}$.

Note. According to this Lemma the tensor $\nabla^{\alpha} K_{\omega \mu \alpha}$ appears only once in $(7.2 \mathrm{~b})$ and it is multiplied by $\frac{1}{2}$.

We shall now investigate the tensor $R_{(\mu \lambda)}$.

Theorem $(7.2 a)$. We have

$$
R_{(\mu \lambda)}=H_{\mu \lambda}+S_{\alpha \mu}{ }^{\beta} S_{\lambda \beta}{ }^{\alpha}-\left(S_{\beta}+U_{\beta}\right) U_{\lambda \mu}^{\beta}
$$

$$
-\nabla_{\alpha} U_{\lambda \mu}^{\alpha}+U_{\alpha \mu}^{\beta} U_{\beta \lambda}^{\alpha}+\nabla_{(\mu}\left(U_{\lambda)}+S_{\lambda)}\right) .
$$

Proof. This equation follows at once from (6.1a, b), (1.2), (7.1) and (1.9).

In the next theorem we use the following abbreviation

$$
\begin{aligned}
& -B_{\mu \lambda}=-B_{(\mu \lambda)}=-\nabla_{\alpha} U_{\lambda \mu}^{\alpha}+U_{\alpha \mu}^{\beta} U_{\beta \lambda}^{\alpha}+\nabla_{\mu} U_{\lambda}-U_{\beta} U_{\lambda \mu}^{\beta} \\
& -\left(K_{\lambda \beta}{ }^{\alpha} U_{\gamma[\mu}^{\beta}+K_{\mu \beta}{ }^{\alpha} U_{\gamma[\lambda}^{\beta}\right) k_{\alpha]}^{\gamma}+4 U_{\gamma[\mu}^{\beta} k_{\alpha]}^{\gamma} U_{\delta[\beta}^{\alpha} k_{\lambda]}{ }^{\delta} .
\end{aligned}
$$

Theorem (7.2b). Equation (7.5a) is equivalent to

$$
R_{(\mu \lambda)}=H_{\mu \lambda}-\frac{1}{4} K_{\alpha \mu}{ }^{\beta} K_{\beta \lambda}{ }^{\alpha}-B_{\mu \lambda}+\nabla_{(\mu} S_{\lambda)}-S_{\beta} U_{\lambda \mu}^{\beta} .
$$

Proof. Substituting from (1.11b) into (7.5a) one obtains (7.5b).

Note. As in the previous theorem, only the last two terms may be looked upon as depending on $S_{\lambda \mu}{ }^{\nu}$. Moreover, the following Lemma holds.

Lemma (7.2). The tensor $B_{\mu \lambda}$ does not contain the term $K_{\alpha \mu}{ }^{\beta} K_{\beta \lambda}{ }^{\alpha}$.

Proof. The solution $U_{\alpha \beta \gamma}$ of (1.13a) is of the form

$$
U_{\alpha \beta \gamma}=Z_{\alpha \beta \gamma}^{\mu \lambda \nu} k_{(\mu}{ }^{\xi} K_{\lambda) \nu \xi},
$$

where $Z$ is a tensor depending on the $k_{\lambda}{ }^{\nu}$ alone (and not on their derivatives). Substituting from (7.7) into (7.6) one cannot obtain the term $K_{\alpha \mu}{ }^{\beta} K_{\beta \lambda}{ }^{\alpha}$, which depends on quadratic products of the derivatives $\nabla_{\alpha} k_{\beta \gamma}$ alone and not on $k_{\lambda}{ }^{\nu}$. Note. According to this lemma the tensor $R_{(\mu \lambda)}$ contains only one term $K_{\alpha \mu}{ }^{\beta} K_{\beta \lambda}{ }^{\alpha}$ and it is multiplied by $-\frac{1}{4}$.

8. A particular case of the field $k_{\lambda \mu}$. In this section we shall investigate first the field $K_{\omega \mu \nu}$. 
Theorem (8.1). A necessary and sufficient condition for

$$
\Gamma_{\lambda \mu}^{\nu}=\left\{\begin{array}{c}
\nu \\
\lambda \mu
\end{array}\right\}
$$

is

$$
K_{\omega \mu \nu}=0 .
$$

Proof. According to (1.8a, b) the system (1.11a) admits only one solution $S_{\omega \mu \nu}$. Hence a necessary and sufficient condition for

$$
S_{\omega \mu \nu}=0
$$

is (8.2). On the other hand we see from (1.9) and (1.10a, b) that a necessary and sufficient condition for (8.1) is (8.3). The proof follows from these facts.

Agreement (8.1). Throughout this paper we shall assume

$$
K_{\omega \mu \nu} \neq 0 .
$$

Note. If (8.4) is satisfied then the requirement

$$
k_{(\rho}{ }^{\alpha} K_{\omega) \mu \alpha}=0
$$

is not an identity. In this section we shall deal with the requirement (8.5) in connection with the field

$$
k_{\mu \lambda}=2 E_{[\mu} u_{\lambda]}
$$

where the vectors $E_{\mu}, u_{\lambda}$ satisfy the following conditions:

$$
E^{\lambda} E_{\lambda}=E_{\lambda} u^{\lambda}=u_{\lambda} u^{\lambda}+1=0^{*} \text {. }
$$

Theorem (8.2). The set of all fields (8.6) for which
(8.7) a) $\quad \nabla_{\mu} E_{\lambda}=\alpha E_{\mu} E_{\lambda}$,
b) $u^{\alpha} \nabla_{\alpha} u_{\lambda}=\beta E_{\lambda}$,
c) $E^{\alpha} \nabla_{\alpha} u_{\lambda}=\gamma E_{\lambda}$
$(\alpha, \beta, \gamma$ arbitrary)

is not an empty set.

Proof. Let $a \neq 0, b \neq 0$ be two arbitrary real functions of the argument $Z \equiv$ $x^{3}-x^{4}$. Put $\varphi \equiv \sqrt{a^{2}+b^{2}}$ and consider the field (8.6a) with
a)$$
E_{\mu}(0,0,1,-1) \text {, }
$$
b)

$$
u_{\lambda}\left(-\frac{b}{\varphi}, \frac{a}{\varphi}, 0,0\right) \text {, }
$$
c)

$$
h_{\lambda \mu}=\left(\left(\begin{array}{cccc}
-1 & 0 & 0 & 0 \\
0 & -1 & 0 & 0 \\
0 & 0 & -1 & 0 \\
0 & 0 & 0 & 1
\end{array}\right)\right) \text {, }
$$

\footnotetext{
* Comparing (8.6) with (1.3) we see that our field is of the third class.
} 
so that (8.6b) are satisfied and all Christoffel symbols $\left\{\begin{array}{c}\nu \\ \lambda \mu\end{array}\right\}$ vanish. Then we have*

$$
\nabla_{\mu} E_{\lambda}=0, \quad \nabla_{\mu} u_{\lambda}=\delta_{\mu}^{3} \partial_{3} u_{\lambda}+\delta_{\mu}^{4} \partial_{4} u_{\lambda}=u_{\lambda}^{\prime}\left(\delta_{\mu}^{3}-\delta_{\mu}^{4}\right)=u_{\lambda}^{\prime} E_{\mu} .
$$

Hence

$$
u^{\alpha} \nabla_{\alpha} u_{\lambda}=u_{\lambda}^{\prime} u^{\mu} E_{\mu}=0, \quad E^{\alpha} \nabla_{\alpha} u_{\lambda}=E^{\alpha} E_{\alpha} u_{\lambda}^{\prime}=0,
$$

and consequently the field $k_{\mu \lambda}$ defined by (8.6) and (8.8) satisfies the conditions (8.7) (with $\alpha=\beta=\gamma=0$ ).

Theorem (8.3). The field $k_{\mu \lambda}$ satisfying (8.6) and (8.7) satisfies also (8.5).

Proof. First of all we have according to (8.6) and (8.7)

$$
k_{\rho}{ }^{\nu} \nabla_{\nu} k_{\omega \mu}=\left(E_{\rho} u^{\nu}-E^{\nu} u_{\rho}\right) \nabla_{\nu}\left(E_{\omega} u_{\mu}-E_{\mu} u_{\omega}\right)=0 .
$$

Moreover,

$$
\begin{aligned}
k_{\rho}^{\beta} \nabla_{[\omega} k_{\beta \mu]} & =2 k_{\rho}^{\beta} \nabla_{[\omega} E_{\beta} u_{\mu]} \\
& =2\left(E_{\rho} u^{\beta}-u_{\rho} E^{\beta}\right) E_{[\beta} \nabla_{\omega} u_{\mu]}=0 .
\end{aligned}
$$

Equations (8.9) together with (1.12) yield easily (8.5).

Theorem (8.4). The field $k_{\lambda \mu}$ defined by (8.6) and (8.7) satisfies the condition

$$
U_{\lambda \mu}^{\nu}=0 \text {. }
$$

Proof. According to Theorem (9.2a) in [6] condition (8.5) is a necessary and sufficient one for (8.10).

Note $I$. The field $k_{\lambda \mu}$ under consideration being of the third class, we have $k=0$ and consequently $P_{\mu}=0(c f r$. (5.4)). On the other hand we obtain $U_{\mu}=0$ from (8.10) so that $(5.3 \mathrm{~b})$ reduces to

$$
\nabla^{\alpha} k_{\alpha \lambda}=0
$$

or

$$
0=\nabla^{\alpha}\left(E_{\alpha} u_{\lambda}-E_{\lambda} u_{\alpha}\right)=\left(\gamma-\nabla_{\alpha} u^{\alpha}\right) E_{\lambda} .
$$

Hence, equation (5.2a) is in our case equivalent to

$$
\gamma=\nabla_{\alpha} u^{\alpha}
$$

In the next paper we shall deal in detail with the fields $k_{\lambda \mu}$ satisfying (8.10).

9. Compatibility problem. In the Appendix II of his book [1] Professor Einstein considers (among others) a system composed of (1.7) and
(9.1) a)
$S_{\lambda}=0$,
b)
$P_{\lambda \mu}=0$.

* The prime indicates the derivative with respect to $Z$. 
He calls this system (I). In a "Note added in proof" he provides an additional argument "for the contention that the choice of system (I) is ... a natural one." On the other hand he states (p. 150) that it is "not at all obvious that the manifold of the solutions of the system (I) is extensive enough to satisfy the requirements of a physical theory ("compatibility") and continues: "In spite of considerable efforts, I did not succeed in proving the compatibility of system (I)".

In this section we shall prove that the system (I) is compatible in the mathematical sense. The proof will be achieved by exhibiting a particular solution $\Gamma_{\lambda \mu}^{\nu}, g_{\lambda \mu}$ of (I). To this effect we introduce the "polar variables"

$$
x^{1} \equiv r, \quad x^{2} \equiv \theta, \quad x^{3} \equiv \varphi, \quad x^{4} \equiv t, \quad 0 \leqq \theta \leqq \pi
$$

as they are used in physical papers and consider the tensor

$$
\left(\left(g_{\lambda \mu}\right)\right) \equiv\left(\left(\begin{array}{cccc}
-1 & 0 & 0 & k_{14} \\
0 & -r^{2} & 0 & 0 \\
0 & 0 & -r^{2} \sin ^{2} \theta & 0 \\
k_{41} & 0 & 0 & h_{44}
\end{array}\right)\right)
$$

where $k_{14} \neq 0, h_{44}>0$ and $k_{14}\left(=-k_{41}\right)$ as well as $h_{44}$ are assumed to be functions of $r$ alone. From (9.2a) we have

(9.2) b) $\mathfrak{h}=-r^{4} h_{44} \sin ^{2} \theta, \quad \mathfrak{g}=X r^{4} \sin ^{2} \theta, \quad g=-X / h_{44} \quad\left(X=k_{14}^{2}-h_{44}\right)$ and the Christoffel symbols of $h_{\lambda_{\mu}}=g_{(\lambda \mu)}$

$(9.2) \mathrm{c})$

$$
\begin{gathered}
\left\{\begin{array}{c}
1 \\
22
\end{array}\right\}=-r, \quad\left\{\begin{array}{c}
1 \\
33
\end{array}\right\}=-r \sin ^{2} \theta, \quad\left\{\begin{array}{c}
1 \\
44
\end{array}\right\}=\frac{1}{2} h_{44}^{\prime}, * \\
\left\{\begin{array}{c}
2 \\
33
\end{array}\right\}=-\sin \theta \cos \theta, \\
\left\{\begin{array}{c}
2 \\
12
\end{array}\right\}=\left\{\begin{array}{c}
3 \\
13
\end{array}\right\}=u \quad\left\{\begin{array}{c}
4 \\
14
\end{array}\right\}=\frac{1}{2}\left(\ln h_{44}\right)^{\prime}, \quad\left\{\begin{array}{c}
3 \\
23
\end{array}\right\}=\operatorname{cotg} \theta \\
\text { the remaining }\left\{\begin{array}{c}
\nu \\
\lambda \mu
\end{array}\right\}=0 .
\end{gathered}
$$

Theorem (9.1). The condition (9.1a) is equivalent to either one of the conditions

$$
r^{4} k_{14}^{2}=X A \quad(A=\text { const., } \operatorname{sgn} X=\operatorname{sgn} A)
$$

b)

$$
\frac{k_{14}^{\prime} h_{44}-\frac{h_{44}^{\prime} k_{14}}{2}}{X}=2 u k_{14}=\frac{h_{44}}{2 k_{14}}(\ln g)^{\prime} \text {. }
$$

* The prime denotes the derivative with respect to $r$. Moreover, we put here and subsequently $u=1 / r$. 
Proof. Comparing $\mathfrak{F}^{\lambda v}$ with the tensor density $\mathfrak{g}^{[\lambda \nu]}$ involved in Theorem (7.3) in [6] we see that (9.1a) is equivalent to (5.2). On the other hand we see from (9.2a) that $\mathfrak{f}=0$ so that $(3.7 \mathrm{a})$ reduces to

$$
\mathfrak{F}^{\lambda \nu}=\frac{\mathfrak{h}}{\sqrt{|\mathfrak{g}|}} k^{\lambda \nu}
$$

e.g.

$$
\mathfrak{F}^{14}=-\mathfrak{F}^{41}=\frac{r^{2} k_{14} \sin \theta}{\sqrt{|X|}}
$$

c)

$$
\text { the remaining } \mathfrak{F}^{\lambda \nu}=0 \text {. }
$$

Hence requirement (9.1a) amounts to the condition

$$
0=\partial_{\lambda} \mathfrak{F}^{\lambda_{\nu}}=\delta_{4}^{\nu}\left(\mathfrak{F}^{14}\right)^{\prime}=\delta_{4}^{\nu}\left(\frac{r^{2} k_{12}}{\sqrt{|X|}}\right)^{\prime} \sin \theta=0
$$

equivalent to (9.3a). Eliminating the constant $A$ from (9.3a) and its derivative, we obtain (9.3b).

Next we need to know the tensor $K_{\omega \mu \nu}$ as given by (1.12). According to (9.2a) we have

$$
\nabla_{[\omega} k_{\nu \mu]}=\partial_{[\omega} k_{\nu \mu]}=0,
$$

so that by virtue of $(1.12)$

$$
K_{\omega \mu \nu}=2 \nabla_{\nu} k_{\omega \mu} .
$$

A straightforward computation based on (9.2c) leads to

$$
\left\{\begin{array}{l}
K_{34 \nu}=-K_{43 \nu}=2\left(r \sin ^{2} \theta \delta_{\nu}^{3}-\frac{1}{2} h_{44}^{\prime} \delta_{\nu}^{4}\right) k_{14} \\
K_{141}=-K_{411}=2\left(k_{14}^{\prime}-\frac{h_{44}^{\prime} k_{14}}{2 h_{44}}\right)=\frac{4 u X k_{14}}{h_{44}} \\
K_{242}=-K_{422}=r k_{14} \\
\text { the remaining } K_{\omega \mu \nu}=0 .
\end{array}\right.
$$

This result will be used in the proof of the next

Theorem (9.2). The tensors $S_{\lambda \mu}{ }^{\nu}$ and $U^{\nu}{ }_{\lambda \mu}$ belonging to the tensor (9.2a) have the components

$$
\left\{\begin{array}{l}
S_{14}{ }^{1}=-S_{41}{ }^{1}=2 u k_{14}=\frac{h_{44}}{2 k_{14}}(\ln g)^{\prime} \\
S_{24}{ }^{2}=-S_{42}{ }^{2}=S_{34}{ }^{3}=-S_{43}{ }^{3}=-u k_{14}=-\frac{h_{44}}{4 k_{14}}(\ln g)^{\prime} \\
\text { the remaining } S_{\lambda \mu}{ }^{\nu}=0
\end{array}\right.
$$


and

$$
\left\{\begin{array}{l}
U^{1}{ }_{44}=4 u k_{14}^{2}=h_{44}(\ln g)^{\prime} \\
U^{4}{ }_{14}=U_{41}^{4}=2 u k_{14}^{2} / h_{44}=\frac{1}{2}(\ln g)^{\prime} \\
\text { the remaining } U_{\lambda \mu}^{v}=0 .
\end{array}\right.
$$

Proof. If we put $\omega=1, \mu=4, \nu=1$ in (1.11a) we obtain

$2 S_{141}=\frac{K_{141}}{1-k_{1}{ }^{4} k_{4}{ }^{1}}=\frac{K_{141}}{1+k_{14} k_{41} h^{44}}=-\frac{-K_{141} h_{44}}{X}=-\frac{4 u X k_{14} h_{44}}{h_{44} X}=-4 u k_{14}$, so that

$$
S_{14}{ }^{1}=2 u k_{14}
$$

The remaining equations (9.7) may be obtained in a similar manner. The equations (9.7) and (1.10b) lead to (9.8).

Theorem (9.3). The solution $\Gamma_{\lambda \mu}^{\nu}$ of (1.7) for the tensor (9.2) is give by

$$
\left\{\begin{array}{l}
\Gamma_{22}^{1}=-r, \quad \Gamma_{33}^{1}=-r \sin ^{2} \theta, \quad \Gamma_{44}^{1}=h_{44}\left(\ln X / \sqrt{h_{44}}\right)^{\prime}=h_{44}\left(\ln g \sqrt{h_{44}}\right)^{\prime} \\
\Gamma_{33}^{2}=-\sin \theta \cos \theta \\
\Gamma_{12}^{2}=\Gamma_{21}^{2}=\Gamma_{13}^{3}=\Gamma_{31}^{3}=u \\
\Gamma_{14}^{4}=\Gamma_{41}^{4}=\frac{1}{2}(\ln X)^{\prime}=\frac{1}{2}\left(\ln g h_{44}\right)^{\prime} \\
\Gamma_{23}^{3}=\Gamma_{32}^{3}=\operatorname{cotg} \theta \\
\Gamma_{14}^{1}=-\Gamma_{41}^{1}=-2 \Gamma_{24}^{2}=+2 \Gamma_{42}^{2}=-2 \Gamma_{34}^{3}=2 \Gamma_{43}^{3}=2 u k_{14}=\frac{h_{44}}{2 k_{14}}(\ln g)^{\prime} \\
\text { the remaining } \Gamma_{\lambda \mu}^{\nu}=0 .
\end{array}\right.
$$

The proof follows by straightforward computation from (1.9), (9.2c), (9.7) and (9.8).

After having found the solution $\Gamma_{\lambda \mu}^{\nu}$ of (1.7) (and (9.1a)) we look for the solution $g_{\lambda \mu}$ of $(9.1 \mathrm{~b})$.

Theorem (9.4). If (9.1a) holds, then (9.1b) is equivalent to

$$
R_{\mu \lambda}=0 .
$$

The proof follows at once from (6.6).

Theorem (9.5). The system

$$
R_{[\lambda \mu]}=0
$$

for the $\Gamma_{\lambda \mu}^{\nu}$ as given by (9.9) is equivalent to

$$
\left(2 u k_{14}\right)^{\prime}+6 u^{2} k_{14}=0 .
$$


Proof. According to (9.1a) we obtain $V_{\omega \mu}=0$ from (6.4b), so that (6.4c) reduces to

$$
R_{[\lambda \mu]}=D_{\alpha} S_{\lambda \mu}^{\alpha} \text {. }
$$

Substituting in this equation for the $\Gamma_{\lambda \mu}^{\nu}$ from (9.9) we obtain

$$
\text { a) } \quad R_{[14]}=\left(2 u k_{14}\right)^{\prime}+6 u^{2} k_{14}
$$

b) the remaining $R_{[\lambda \mu]}=0$.

Theorem (9.6). Equation (9.11) yields

$$
R_{(\lambda \mu)}=0 .
$$

Proof. The solution of (9.11) is by virtue of (9.3)

$$
X=\text { const. }
$$

On the other hand one obtains from (9.9)

$$
R_{22}=\frac{r}{2}(\ln X)^{\prime}=0 .
$$

Substituting (9.14) into (9.9) one obtains easily

$$
R_{(\lambda \mu)}=0 .
$$

Theorem (9.7). A solution $g_{\lambda \mu}$ of (1.7) and (9.1) is given by (9.2a), where

$$
\begin{array}{ll}
\text { a) } & k_{14}=\epsilon \sqrt{|B A|} u^{2}, \\
\text { b) } & h_{44}=B\left(1-A u^{4}\right) .
\end{array}
$$

Here $B$ is a positive constant, $A$ is a negative constant and $\epsilon= \pm 1$.

Proof. According to the second equation of $(9.2 \mathrm{~b})$ and by virtue of $(1.8 \mathrm{c}, \mathrm{d})$ and (9.14) $X$ must be a negative constant which we denote by $X=-B$ where $B$ is a positive constant. Then we have from (9.3a)

$$
k_{14}^{2}=-A B u^{4}=|A B| u^{4}
$$

for $\operatorname{sgn} A=\operatorname{sgn}(-B)$. This equation yields (9.16a), while $X=k_{14}^{2}-h_{44}$ leads to $(9.16 \mathrm{~b})$.

Theorem (9.8). The solution mentioned in Theorem (9.7) is also a solution of (1.7), (9.1a) and

$$
P_{(\lambda \mu)}=0, \quad \partial_{[\omega} P_{\mu \lambda]}=0 .
$$

The proof is obvious.*

\footnotetext{
* For an (incomplete) bibliography of solutions of this weaker system $c f r$. [7].
} 


\section{Chapter II}

1. Introduction.* In this chapter we shall investigate the solutions of a system which consists of the equations

a)

b)

$$
\partial_{\omega} g_{\lambda \mu}=\Gamma_{\lambda \omega}^{\alpha} g_{\alpha \mu}+\Gamma_{\omega \mu}^{\alpha} g_{\lambda \alpha}
$$

$$
S_{\lambda}=0
$$

and either

$$
R_{\mu \lambda}=0
$$

or
(1.3) a)
$R_{(\mu \lambda)}=0$,
b)
$\partial_{[\omega} R_{\mu \lambda]}=0$.

We shall replace (1.1) by an equivalent system

a)

b)

$$
D_{\omega} g_{\lambda \mu}=2 S_{\omega \mu}^{\alpha} g_{\lambda \alpha}
$$

$$
S_{\lambda}=0
$$

and condense the conditions (1.2) and (1.3) into one:

$$
R_{\mu \lambda}=\partial_{[\mu} X_{\lambda]}
$$

If dealing with (1.2) we have to assume that $X_{\lambda}$ is an arbitrary gradient vector, while in the case (1.3) $X_{\lambda}$ is an arbitrary vector. This latter case will be referred to as a general case while the case of a gradient vector $X_{\lambda}$ will be termed a special case.

The main problems dealt with may be characterized as follows:

1) To find the most general solution $g_{\lambda \mu}$ of (1.4) for a given connection $\Gamma_{\lambda \mu}^{\nu}$.

2) To find the most general solution $\Gamma_{\lambda \mu}^{\nu}$ of (1.5).

3) To find the most general solution $g_{\lambda \mu}$ of (1.4) and (1.5).

Equations (1.1) and (1.2) (or (1.1) and (1.3)) have been devised by Einstein [1], [2] in order to get $g_{\lambda \mu}$. The usual procedure of "solving" this system may be described as follows: One considers first $g_{\lambda \mu}$ as auxiliary variables and solves (1.1) for $\Gamma_{\lambda \mu}^{\nu}$ in terms of $g_{\lambda \mu}$. Upon substituting this solution in (1.2) (in (1.3)) one obtains sixteen partial differential equations of the second order for the $g_{\lambda \mu}$ (ten partial differential equations of the second order and four partial differential equations of the third order for the $\left.g_{\lambda \mu}\right)$. Although this system may be solved in

* Whenever we use results from the first chapter we denote them by $\mathrm{I}$. Thus for instance Theorem $I(6.4)$ refers to Theorem (6.4) in the first chapter. 
special cases (cfr. for instance the Section I 9) it is "next to impossible" to solve it without additional restrictions.

Therefore we devise here another method. First we find the most general solution $\Gamma_{\lambda \mu}^{\nu}$ of (1.5) in terms of a particular solution. This requires sixteen partial differential equations of the first order only. Then the corresponding $g_{\lambda \mu}$ may be found from (1.4) by means of a method which in some cases is purely algebraic and enables us to find all $g_{\lambda \mu}$ belonging to the given $\Gamma_{\lambda \mu}^{\nu}$.

Some less important problems are also dealt with in this section, for instance the problem of conformal change of $g_{\lambda \mu}$, and so on.

2. Conformal change of $g_{\lambda \mu}$. Let $\rho \neq 0$ be a real scalar function of the coordinates. The transformations ' $g_{\lambda \mu} \rightarrow g_{\lambda \mu}$ given by

$$
{ }^{\prime} g_{\lambda \mu}=\rho^{2} g_{\lambda \mu}
$$

will be termed a conformal change (of $g_{\lambda \mu}$ ).

Theorem (2.1). The most general conformal change admitted by (1.4)* which does not change the $\Gamma_{\lambda \mu}^{\nu}$ is (2.1) where $\rho=$ const.

Proof. If (2.1) and

$$
{ }^{\prime} \Gamma_{\lambda \mu}^{\nu}=\Gamma_{\lambda \mu}^{\nu}
$$

hold and if (1.4) admits (2.1), then we must have

$$
\begin{aligned}
0 & =D_{\omega}{ }^{\prime} g_{\lambda \mu}-2 S_{\omega \mu}{ }^{\alpha}{ }^{\prime} g_{\lambda \alpha} \\
& =\rho\left\{\left(D_{\omega} \ln \rho\right) g_{\lambda \mu}+D_{\omega} g_{\lambda \mu}-2 S_{\omega \mu}{ }^{\alpha} g_{\lambda \alpha}\right\} \\
& =\left(D_{\omega} \rho\right) g_{\lambda \mu} .
\end{aligned}
$$

This equation yields $\rho=$ const. because $\mathfrak{g} \neq 0$.

Note. According to this theorem the conformal changes (2.1) admitted by (1.4) and satisfying (2.2) are very limited as long as one confines oneself to the scalar function $\rho$. The situation changes if instead of a scalar function $\rho$ we introduce a real density $\mathfrak{r}$

$$
\mathfrak{g}_{\lambda \mu}=\mathfrak{r} g_{\lambda \mu}
$$

Theorem (2.2). The most general real density $\mathfrak{r}$ for which
(2.4) a)
$D_{\omega} \mathfrak{g}_{\lambda \mu}=2 S_{\omega \mu}^{\alpha} \mathfrak{g}_{\lambda \alpha}$,
b)
$S_{\lambda}=0$

* Denote by ${ }^{\prime} \Gamma_{\lambda \mu}^{\nu}$ the solution of

$$
{ }^{\prime} D_{\omega}{ }^{\prime} g_{\lambda \mu}=2{ }^{\prime} S_{\omega \mu}{ }^{\alpha} g_{\lambda \alpha}, \quad{ }^{\prime} S_{\lambda}=0
$$

where ' $g_{\lambda \mu}$ is given by (2.1). If such a solution exists we say that (1.4) admits the conformal change (2.1). 
hold is

$$
\mathfrak{r}=|\mathfrak{g}|^{p} c \quad(c=\text { a real const. }>0),
$$

where $p$ is an arbitrary real number.

Proof. From (2.4) we obtain

$$
0=\left(D_{\omega} \mathfrak{r}\right) g_{\lambda \mu}+\mathfrak{r}\left(D_{\omega} g_{\lambda \mu}-2 S_{\omega \mu}{ }^{\alpha} g_{\lambda \alpha}\right)=\left(D_{\omega} \mathfrak{r}\right) g_{\lambda \mu},
$$

so that for $(2.4 \mathrm{a})$ to be satisfied we must have

$$
D_{\omega} \mathfrak{x}=0 .
$$

On the other hand the condition $(2.4 \mathrm{~b})$ yields

$$
D_{\omega} \mathfrak{g}=0^{*} \text {. }
$$

It may be easily shown from (2.7) that $\mathfrak{x}$ as defined by (2.5) is the only density satisfying (2.6b).

Note. So far we have investigated the cases for which (2.2) holds. A more general case arises when we drop this condition. In order to deal with this more general problem we need a tensor $Y_{\xi \eta \zeta}^{\omega \lambda \nu}=Y_{[\xi \eta] \zeta}^{[\omega \lambda] \nu}$ defined in the following way. First we define the tensor $X_{\omega \mu \nu}^{\xi \eta \xi}=X_{[\omega \mu] \nu}^{[\xi \eta] \xi}$ by

$$
X_{\omega \mu \nu}^{\xi \eta \zeta} \equiv \delta_{[\omega \mu] \nu}^{[\xi] \zeta}-2 \delta_{\nu}^{[\xi} k_{[\omega}^{\eta]} k_{\mu]}^{\zeta}-2 \delta_{[\mu}^{[\xi} k_{\omega]}^{\eta]} k_{\nu}^{\zeta},
$$

so that $I(1.11 a)$ could be written

$$
2 S_{\xi \eta \eta} X_{\omega \mu \nu}^{\xi \eta \xi}=K_{\omega \mu \nu} .
$$

Whenever I(1.8a, b) hold this equation admits exactly one solution $S_{\xi \eta \zeta}(c f r$. [7])

$$
2 S_{\xi \eta \zeta}=K_{(v \mu \nu} Y_{\xi \eta \xi}^{\omega \mu \nu}
$$

so that in this case there is exactly one tensor $Y_{\xi \eta \xi}^{\pi \mu \nu}$ defined by

$$
X_{\alpha \beta \gamma}^{\xi \eta \xi} Y_{\xi \eta \zeta}^{\omega \mu \nu}=\delta_{[\alpha \beta] \gamma}^{[\omega \mu] \nu} \cdot \dagger
$$

This tensor is involved in the next

Theorem (2.3). Necessary and sufficient conditions that (1.4 a, b) admit a conformal change (2.1) are: there is a gradient $r_{\mu}=\partial_{\mu} \ln \rho$ which satisfies the conditions

$$
\begin{aligned}
\text { a) } r^{\delta} Y_{\xi \eta \zeta}^{\alpha \beta \gamma}\left(h_{\delta \gamma} k_{\alpha \beta}\right. & \left.+2 k_{\delta[\alpha} h_{\beta] \gamma}\right) \delta_{\lambda}^{[\xi} h^{\eta] \zeta}=0 \\
\text { (2.11) b) } T_{\lambda \mu}{ }^{\nu}=r_{\lambda} \delta_{\mu}^{\nu}+ & r_{\mu} \delta_{\lambda}^{\nu}-r^{\nu} h_{\mu \lambda} \\
& -r^{\delta} Y_{\xi \eta \zeta}^{\alpha \beta \gamma}\left(h_{\gamma \delta} k_{\alpha \beta}+2 k_{\delta[\alpha} h_{\beta] \gamma}\right)\left(h^{\zeta \nu} \delta_{\lambda \mu}^{\xi \eta}+2 h^{\xi \nu} \delta_{(\lambda}^{\eta} k_{\mu)}^{\zeta}\right),
\end{aligned}
$$

${ }^{*} C f r .[6]$ p. 28.

$\dagger$ The actual computation of $Y$ is given in Theorem (4.4) in [6]. 
where

(2.11) c)

$$
{ }^{\prime} \Gamma_{\lambda \mu}^{\nu}=\Gamma_{\lambda \mu}^{\nu}+T_{\lambda \mu}{ }^{\nu}
$$

is the connection belonging to the tensor ' $g_{\lambda \mu}$ by means of
(2.12) a)
${ }^{\prime} D_{\omega}{ }^{\prime} g_{\lambda \mu}=2{ }^{\prime} S_{\omega \mu}{ }^{\alpha} g_{\lambda \alpha}$,
b)
${ }^{\prime} S_{\lambda}=0$.

Proof. The tensor $X$ is invariant under the conformal change (2.1), which splits into
(2.13) a)
${ }^{\prime} h_{\lambda \mu}=\rho^{2} h_{\lambda \mu}$,
b)
${ }^{\prime} k_{\lambda \mu}=\rho^{2} k_{\lambda \mu}$

Therefore we have from (2.10)

$$
{ }^{\prime} Y_{\xi \eta \zeta}^{\omega \mu \nu}=Y_{\xi \eta \zeta}^{\omega \mu \nu}
$$

A necessary and sufficient condition that (1.4a) admit the conformal change (2.1) is (2.12a). Taking into account (2.14) as well as the equation (4.13) in [6] we see that the solution of $(2.12 \mathrm{a})$ is

$$
\left.{ }^{\prime} \Gamma_{\lambda \mu}^{\nu}=\left\{\begin{array}{c}
\nu \\
\lambda \mu
\end{array}\right\}+\frac{1}{2}^{\prime} K_{\alpha \beta \gamma} Y_{\xi \eta \zeta}^{\alpha \beta \gamma}\left(h^{\zeta \nu} \delta_{\lambda \mu}^{\xi \eta}+2^{\prime} h^{\xi \nu} \delta_{(\lambda}^{\eta}{ }^{\prime} k_{\mu}\right)^{\zeta}\right) .
$$

On the other hand we have from (2.13a)

$$
\left\{\begin{array}{c}
\nu \\
\lambda \mu
\end{array}\right\}=\left\{\begin{array}{c}
\nu \\
\lambda \mu
\end{array}\right\}+r_{\lambda} \delta_{\mu}^{\nu}+r_{\mu} \delta_{\lambda}^{\nu}-r^{\nu} h_{\lambda \mu},
$$

and from $(2.13 \mathrm{a}, \mathrm{b})$ and $\mathrm{I}(1.12)$

$$
' K_{\omega \mu \nu}=\rho^{2}\left\{K_{\omega \mu \nu}-2 r_{\nu} k_{\omega \mu}+4 r^{\alpha} k_{\alpha[\mu} h_{\omega] \nu}\right\} .
$$

Substituting from (2.16) back into (2.15) one obtains $(2.11 b, c)$. From (2.11b) we obtain

$$
T_{[\lambda \alpha]}^{\alpha}=-r^{\delta} Y_{\xi \eta \zeta}^{\alpha \beta \gamma}\left(h_{\delta \gamma} k_{\alpha \beta}+2 k_{\delta[\alpha} h_{\beta] \gamma}\right) h^{\zeta[\eta} \delta_{\lambda}^{\xi]} .
$$

Remembering (2.11c) we have

$$
{ }^{\prime} S_{\lambda}=S_{\lambda}+T_{[\lambda \alpha]}^{\alpha}=T_{[\lambda \alpha]}^{\alpha} .
$$

Equations (2.12b) and (2.17) yield (2.11a). The equations (2.11a, b) are obviously necessary and sufficient for ' $\Gamma_{\lambda \mu}^{\nu}$ given by (2.11c) to be the solution of (2.12).

In the next theorem we use the abbreviation

$$
Z_{\lambda \mu} \equiv Y_{\xi \eta \zeta}^{\alpha \beta \gamma}\left(h_{\gamma \mu} k_{\alpha \beta}+2 k_{\mu[\alpha} h_{\beta] \gamma}\right) \delta_{\lambda}^{[\xi} h^{\eta] \zeta} .
$$

Theorem (2.4). Necessary and sufficient conditions for exactly one conformal change (2.1) leading to (2.12) are: 
1) The tensor $Z_{\lambda \mu}$ is of rank 3 so that there is exactly one solution $x^{\mu *}$ of

$$
x^{\mu} Z_{\lambda \mu}=0
$$

2) There is a gradient $a_{\mu}=\partial_{\mu} \ln \alpha$ such that

$$
x_{[\mu} a_{\lambda]}+\partial_{[\lambda} x_{\mu]}=0 .
$$

If these conditions are satisfied then

$$
r_{\mu}=\alpha x_{\mu}
$$

is a gradient

$$
r_{\mu}=\partial_{\mu} \ln \rho
$$

and $\rho$ is the factor involved in (2.1).

The proof is almost obvious.

3. The most general solution $g_{\lambda \mu}$ of (1.4a). In this section we shall deal with the problem of existence of $g_{\lambda \mu}$ satisfying (1.4a) for an a priori given connection $\Gamma_{\lambda \mu}^{\nu}$ (for instance a solution of (1.5)). This problem is connected with the tensors $V$ defined as follows:

$$
\begin{aligned}
V_{\xi_{1} \omega \lambda \mu}^{\alpha \beta} \equiv & \frac{1}{2} R_{\xi_{1} \omega \lambda}{ }^{\alpha} \delta_{\mu}^{\beta} \\
& \quad+\delta_{\lambda}^{\alpha}\left[\frac{1}{2} R_{\xi_{1} \omega \mu}{ }^{\beta}+2 S_{\xi_{1} \omega}{ }^{\gamma} S_{\gamma \mu}{ }^{\beta}-2 D_{\left[\xi_{1}\right.} S_{\omega] \mu}{ }^{\beta}+4 S_{\mu[\omega}{ }^{\gamma} S_{\left.\xi_{1}\right] \gamma}{ }^{\beta},\right.
\end{aligned}
$$

(3.1) b) $\quad V_{\xi_{p+1} \cdots \xi_{1} \omega \lambda \mu}^{\alpha \beta} \equiv D_{\xi_{p+1}} V_{\xi_{p} \cdots \xi_{1} \omega \lambda \mu}^{\alpha \beta}+2 S_{\xi_{p+1 \gamma}{ }^{\beta}}^{\alpha \beta} V_{\xi_{p} \cdots \xi_{1} \omega \lambda \mu}^{\alpha \gamma}, \quad p=1,2, \cdots$

Moreover, we denote by $((V))_{p}$ the matrix of 16 columns whose elements are $V_{\xi_{p} \cdots \xi_{1} \omega \lambda \mu}^{\alpha \beta}$. The columns of this matrix are labeled by superscripts $\alpha \beta$, while the rows are labeled by the subscripts $\xi_{p} \cdots \xi_{1} \omega \lambda \mu . \dagger$

Theorem (3.1). A necessary and sufficient condition that the system (1.4a) admit exactly $q(<16)$ independent solutions ${ }^{1} g_{\lambda \mu}, \cdots,{ }^{q} g_{\lambda \mu}$ (for a given system of $\left.\Gamma_{\lambda \mu}^{\nu}\right)$ is: there is a positive integer $N \geqq 1 \ddagger$ such that for $p \geqq N$ the rank of $((V))_{p}$ is $16-q$. If this condition is satisfied then there exists a set of $q$ linearly independent solutions ${ }^{1} x_{\lambda \mu}, \cdots{ }^{q} x_{\lambda \mu}$ of the algebraic system

$$
x_{\alpha \beta} V_{\xi_{r} \cdots \xi_{1} \omega \lambda \mu}^{\alpha \beta}=0, \quad \quad r=1,2, \cdots N
$$

and $a$ set of scalars ${ }^{1} \alpha, \cdots,{ }^{q} \alpha$ such that the system

$$
{ }^{i} a_{\omega}{ }^{i} x_{\lambda \mu}+D_{\omega}{ }^{i} x_{\lambda \mu}-2 S_{\omega \mu}{ }^{\alpha}{ }^{i} x_{\lambda \alpha}=0 \quad\left({ }^{i} a_{\omega}=\partial_{\omega} \ln { }^{i} \alpha, i=1, \cdots, q\right)
$$

* Up to a factor of proportionality.

$\dagger$ The set of the first $4^{4}$ rows are labeled by $\xi_{1} \omega \lambda \mu$, the next set of $4^{5}$ rows by $\xi_{2} \xi_{1} \omega \lambda \mu_{\text {, }}$ and so on.

$\ddagger$ We must obviously have $N \leqq 15$. 
is a completely integrable one and

$$
{ }^{i} g_{\lambda \mu}={ }^{i} \alpha{ }^{i} x_{\lambda \mu}
$$$$
i=1, \cdots, q
$$

are the solutions of $(1.4 a)$.

Proof. The integrability conditions for (1.4a) may be obtained from

$$
D_{[\xi} D_{\omega]} g_{\lambda \mu}=2 D_{[\xi} S_{\omega] \mu}^{\beta} g_{\lambda \beta},
$$

where into the left hand term we substitute from the well known identity of the type $\mathrm{I}(6.2)$ and eliminate on both sides the covariant derivative of $g_{\lambda \mu}$ by means of (1.4a). In doing so we obtain

$$
g_{\alpha \beta} V_{\xi \omega \lambda \mu}^{\alpha \beta}=0
$$

where $V_{\xi \omega \lambda \mu}^{\alpha \beta}$ is defined by (3.1a). Taking the covariant derivative of this equation, eliminating the covariant derivative of $g_{\lambda \mu}$ by means of (1.4a) and repeating this procedure we obtain finally a set of algebraic equations

$$
g_{\alpha \beta} V_{\xi_{p} \cdots \xi_{1} \omega \lambda \mu}^{\alpha \beta}=0
$$$$
p=1,2, \cdots
$$

The above stated theorem is nothing else than an application of a well known existence theorem* to (1.4a) and $(3.6)_{p}$.

Note $I$. The importance of this theorem lies in the possibility of deciding by purely algebraic means whether the system (1.4) admits solutions. In the positive case $16-q$ components of ${ }^{i} x_{\lambda \mu}$ are obtained by purely algebraic operations.

Note II. Any linear combination with constant coefficients of the tensors (3.4) is also a solution of (1.4a).

Theorem (3.2). A necessary and sufficient condition that ${ }^{i} g_{\lambda \mu}$ be of rank 4 is

$$
\mathfrak{G}^{\alpha \beta \gamma \hat{0}}\left(^{\omega \mu \lambda \nu}{ }^{i} x_{\alpha \omega}{ }^{i} x_{\beta \mu}{ }^{i} x_{\gamma \lambda}{ }^{i} x_{\delta \nu} \neq 0 .\right.
$$

Proof. Multiplying the left hand side of (3.7) by $\left({ }^{i} \alpha\right)^{4}$ one obtains up to a numerical multiplicative factor the determinant of ${ }^{i} g_{\lambda \mu}$.

4. The most general solution $\Gamma_{\lambda \mu}^{\nu}$ of (1.5). In the previous section we dealt with the most general solution of (1.4a) where $\Gamma_{\lambda \mu}^{\nu}$ were assumed to be known. In this section we shall find the most general solution $\Gamma_{\lambda \mu}^{\nu}$ of (1.5). The corresponding considerations are based on the following auxiliary

Theorem (4.1). The transformation

$$
{ }^{*} \Gamma_{\lambda \mu}^{\nu}=\Gamma_{\lambda \mu}^{\nu}+T_{\lambda \mu}^{\nu}
$$

\footnotetext{
* See for instance [3] p. 18.
} 
changes $R_{\mu \lambda}$ into

$$
\begin{aligned}
{ }^{*} R_{\mu \lambda}=R_{\mu \lambda}- & \left(D_{\alpha} T_{\lambda \mu}{ }^{\alpha}+T_{\alpha} T_{\lambda \mu}{ }^{\alpha}\right) \\
& +D_{\mu} T_{\lambda}+2 S_{\alpha \mu}{ }^{\beta} T_{\lambda \beta}{ }^{\alpha}+T_{\beta \mu}{ }^{\alpha} T_{\lambda \alpha}{ }^{\beta} \quad\left(T_{\alpha} \equiv T_{\lambda \alpha}{ }^{\alpha}\right) .
\end{aligned}
$$

Proof. One obtains (4.1b) by substituting from (4.1a) into

$$
{ }^{*} R_{\mu \lambda}=\partial_{\mu} * \Gamma_{\lambda \alpha}^{\alpha}-\partial_{\alpha} * \Gamma_{\lambda \mu}^{\alpha}+{ }^{*} \Gamma_{\beta \mu}^{\alpha} * \Gamma_{\lambda \alpha}^{\beta}-{ }^{*} \Gamma_{\alpha \beta}^{\beta} * \Gamma_{\lambda \mu}^{\alpha} .
$$

Note. It is not difficult to find a solution $\Gamma_{\lambda \mu}^{\nu}$ for which (1.4b) does not hold. Consider for instance the connection

$$
\Gamma_{\lambda \mu}^{\nu}=\left\{\begin{array}{c}
\nu \\
\lambda \mu
\end{array}\right\}+\delta_{\lambda}^{\nu} s_{\mu},
$$

where $\left\{\begin{array}{c}\nu \\ \lambda \mu\end{array}\right\}$ are Christoffel symbols belonging to an arbitrary tensor $h_{\lambda \mu}=h_{(\lambda \mu)}$ and $s_{\mu} \neq 0$ is a gradient in the special case. Denoting by $H_{\mu \lambda}$ its contracted curvature tensor we obtain easily

$$
R_{\mu \lambda}=H_{\mu \lambda}+2 \partial_{[\mu} s_{\lambda]} .
$$

Hence if we take for $h_{\lambda \mu}$ the tensor whose contracted curvature tensor $H_{\lambda \mu}$ vanishes (for instance the Schwarzschild solution) then (4.2a) is a solution of (1.5b) with $S_{\lambda}=-\frac{3}{2} s_{\lambda} \neq 0$.

In the subsequent considerations we assume that we know already a solution of (1.5) which we denote by ${ }^{0} \Gamma_{\lambda \mu}^{\nu}$. All objects referring to this particular solution will be labeled by 0 .

Theorem (4.2). Let ${ }^{0} \Gamma_{\lambda \mu}^{\nu}$ be a solution of (1.5). The most general solution of (1.5) and (1.4b) is

$$
\Gamma_{\lambda \mu}^{\nu}={ }^{0} \Gamma_{\lambda \mu}^{\nu}+T_{\lambda \mu}^{\nu}
$$

where the tensor $T_{\lambda \mu}{ }^{\nu}$ satisfies the conditions

a) ${ }^{0} S_{\lambda}+T_{[\lambda \alpha]}^{\alpha}=0$,

b) $\partial_{[\mu} X_{\lambda]}={ }^{0} D_{\mu} T_{\lambda}+2{ }^{0} S_{\alpha \mu}{ }^{\beta} T_{\lambda \beta}{ }^{\alpha}+T_{\beta \mu}{ }^{\alpha} T_{\lambda \alpha}{ }^{\beta}-\left({ }^{0} D_{\alpha} T_{\lambda \mu}{ }^{\alpha}+T_{\alpha} T_{\lambda \mu}{ }^{\alpha}\right)$.

Here $X_{\mu}$ is an arbitrary vector (arbitrary gradient) in the general (special) case.* The set of all tensors $T_{\lambda \mu}{ }^{\nu}$ satisfying (4.4) is not an empty set.

Proof. Any connection $\Gamma_{\lambda \mu}^{\nu}$ may be written in the form (4.3). This equation yields

$$
S_{\lambda}={ }^{0} S_{\lambda}+T_{[\lambda \alpha]}^{\alpha}
$$

\footnotetext{
* This vector is not necessarily the same as $X_{\mu}$ involved in (1.5).
} 
so that we obtain (1.4b) if and only if (4.4a) holds. The statement about (4.4b) follows at once from the previous theorem. The last statement will be proved simultaneously with the following theorem, where we assume that ${ }^{0} S_{\lambda}$ is not a gradient.

Theorem (4.3). Let ${ }^{0} \Gamma_{\mu \lambda}^{\nu}$ be a solution of (1.5) (in the general case) which does not satisfy (1.4b). Then

$$
\Gamma_{\lambda \mu}^{\nu}={ }^{0} \Gamma_{\lambda \mu}^{\nu}+\frac{2}{3}{ }^{0} S_{\mu} \delta_{\lambda}^{\nu}
$$

is a solution of (1.4b) and of (1.5) (in the general case).*

Proof. Denote by $q_{\mu}$ a non gradient, and put

$$
T_{\lambda \mu}{ }^{\nu}=\delta_{\lambda}^{\nu} q_{\mu}, \quad T_{\lambda}=q_{\lambda} .
$$

Then

$$
\begin{aligned}
{ }^{0} D_{\mu} T_{\lambda} & ={ }^{0} D_{\mu} q_{\lambda} \\
2{ }^{0} S_{\alpha \mu}{ }^{\beta} T_{\lambda \beta}{ }^{\alpha} & =2{ }^{0} S_{\alpha \mu}{ }^{\beta} \delta_{\lambda}^{\alpha} q_{\beta}=2{ }^{0} S_{\lambda \mu}{ }^{\alpha} q_{\alpha} \\
T_{\beta \mu}{ }^{\alpha} T_{\lambda \alpha}{ }^{\beta} & =\delta_{\beta}^{\alpha} \delta_{\lambda}^{\beta} q_{\mu} q_{\alpha}=q_{\mu} q_{\lambda} \\
-\left({ }^{0} D_{\alpha} T_{\lambda \mu}{ }^{\alpha}+T_{\alpha} T_{\lambda \mu}{ }^{\alpha}\right) & =-\left({ }^{0} D_{\lambda} q_{\mu}+q_{\lambda} q_{\mu}\right),
\end{aligned}
$$

so that $(4.4 \mathrm{~b})$ reduces to

$$
\partial_{[\mu} X_{\lambda]}=2\left({ }^{0} D_{[\mu} q_{\lambda]}+{ }^{0} S_{\lambda \mu}{ }^{\alpha} q_{\alpha}\right) \equiv 2 \partial_{[\mu} q_{\lambda]} .
$$

Hence $(4.4 \mathrm{~b})$ is satisfied in the general case. On the other hand we have from (4.3) and (4.7)

$$
S_{\lambda}={ }^{0} S_{\lambda}-\frac{3}{2} q_{\lambda}
$$

so that (4.7) with $q_{\lambda}=\frac{2}{3}{ }^{0} S_{\lambda}$ satisfies also (1.4b).

Note. The previous theorem proves the existence of $T_{\lambda \mu}{ }^{\nu}$ which satisfies (4.4) (in the general case at least)* as it was asserted in the last statement of the theorem (4.2).

Theorem (4.4). Let ${ }^{0} \Gamma_{\lambda \mu}^{\nu}$ be a solution of (1.4b) and (1.5). Then (4.3) is the most general solution of this system provided that $T_{\lambda \mu}{ }^{\nu}$ satisfies (4.4b) and

$$
T_{[\lambda \alpha]}^{\alpha}=0 .
$$

The set of all tensors satisfying these conditions is not an empty set.

Proof. The first part of this theorem may be proved in a similar manner as that used in the proof of Theorem (4.2). The last statement will be proved by the following

* It may be shown easily that this theorem holds also for the special case provided that ${ }^{0} S_{\lambda}$ is a gradient. 
Illustration (4.1). Let us start with the solution derived in the Section I 9 and given in Theorem I(9.3) (for $X=$ const.).* Put

$$
T_{\lambda \mu}{ }^{\nu}=k_{\lambda \mu} p^{\nu}=T_{[\lambda \mu]}{ }^{\nu},
$$

so that according to $(4.9)$ we have to have

$$
k_{\lambda \alpha} p^{\alpha}=0 \text {. }
$$

Remembering Theorem I(9.7) we see from (4.11a) that we must have

$$
p^{v}\left(0, p^{2}, p^{3}, 0\right)
$$

and moreover, according to (4.10) and (4.11a),

$$
T_{\lambda}=0 \text {. }
$$

Hence

$$
\begin{aligned}
{ }^{0} D_{\mu} T_{\lambda} & =0, \\
2 S_{\alpha \mu}{ }^{\beta} T_{\lambda \beta}{ }^{\alpha} & =2 S_{\alpha \mu}{ }^{\beta} k_{\lambda \beta} p^{\alpha}=U_{\alpha \mu \lambda} p^{\alpha}+2 S_{\alpha[\mu}{ }^{\beta} k_{\lambda] \beta} p^{\alpha} \\
T_{\beta \mu}{ }^{\alpha} T_{\lambda \alpha}{ }^{\beta} & =k_{\beta \mu} k_{\lambda \alpha} p^{\alpha} p^{\beta}=0 \\
-\left({ }^{0} D_{\alpha} T_{\lambda \mu}{ }^{\alpha}+T_{\alpha} T^{\alpha}{ }^{\alpha}\right) & =-{ }^{0} D_{\alpha} k_{\lambda \mu} p^{\alpha} \\
& =-\left[2 p^{\alpha} S_{\alpha[\mu}{ }^{\beta} g_{\lambda] \beta}+k_{\lambda \mu}{ }^{0} D_{\alpha} p^{\alpha}\right] .
\end{aligned}
$$

Consequently $(4.4 \mathrm{~b})$ reduces to

$$
\begin{aligned}
\partial_{[\mu} X_{\lambda]}=U_{\alpha \mu \lambda} p^{\alpha}+2 S_{\alpha[\mu}{ }^{\beta} & k_{\lambda] \beta} p^{\alpha} \\
& -2 S_{\alpha[\mu \lambda]} p^{\alpha}-2 S_{\alpha[\mu}{ }^{\beta} k_{\lambda] \beta} p^{\alpha}-k_{\lambda \mu}{ }^{0} D_{\alpha} p^{\alpha} .
\end{aligned}
$$

Remembering $I(9.8)$ and (4.12) we see that

$$
U_{\alpha \mu \lambda} p^{\alpha}=0,
$$

so that (4.13a) finally reduces to

$$
-\partial_{[\mu} X_{\lambda]}=2 S_{\alpha[\mu \lambda]} p^{\alpha}+k_{\lambda \mu}^{0} D_{\alpha} p^{\alpha} .
$$

Using $I(9.7)$ as well as (4.12) we may write (4.13) in the following form:
a) $\partial_{[1} X_{2]}=0$
b) $\partial_{[2} X_{3]}=0$,
c) $\partial_{[3} X_{1]}=0$,
e) $\partial_{[3} X_{4]}=S_{343} p^{3}$
f) $\partial_{[1} X_{4]}=k_{14}^{0} D_{\alpha} p^{\alpha}$
g) $\partial_{[2} X_{4]}=S_{242} p^{2}$

\footnotetext{
* Here we write simply $S_{\lambda \mu}{ }^{\nu}, U^{\nu}{ }_{\lambda \mu}, \Gamma_{\lambda \mu}^{\nu}$ instead of ${ }^{0} S_{\lambda \mu}{ }^{\nu},{ }^{0} U^{\nu}{ }_{\lambda \mu},{ }^{0} \Gamma_{\lambda \mu}^{\nu}$.
} 
On the other hand by virtue of $\mathrm{I}(9.9)$

$$
\begin{aligned}
{ }^{0} D_{\alpha} p^{\alpha} & =\partial_{2} p^{2}+\partial_{3} p^{3}+\Gamma_{2 \alpha}^{\alpha} p^{2}+\Gamma_{3 \alpha}^{\alpha} p^{3} \\
& =\partial_{2} p^{2}+\partial_{3} p^{3}+p^{2} \cot \theta
\end{aligned}
$$

Hence if we choose

$$
p^{2}=\frac{a}{\sin \theta}, \quad p^{3}=p^{3}(r, \theta, t) \quad(a=\text { const. }),
$$

we obtain instead of (4.14f)

$$
\partial_{[1} X_{4]}=0 .
$$

If we put $X_{4}=0$, we may rewrite $\left(4.14 \mathrm{e}, \mathrm{f}^{\prime}, \mathrm{g}\right)$ as follows

$$
\begin{array}{rr}
X_{3}=-2 S_{343} \int p^{3} d t+Y_{3}(r, \theta, \varphi), \\
X_{2}=-2 S_{242} p^{2} t+Y_{2}(r, \theta, \varphi), \\
X_{1}= & Y_{1}(r, \theta, \varphi),
\end{array}
$$

so that $(4.14 a, b, c)$ yield

a)

b)

c)

$$
\begin{aligned}
& \partial_{[1} Y_{2]}-S_{242}^{\prime} p^{2} t=0 \\
& \partial_{[2} Y_{3]}-S_{343} \frac{\partial}{\partial \theta} \int p^{3} d t=0
\end{aligned}
$$

$$
\partial_{[3} Y_{1]}+\left(S_{343} \int p^{3} d t\right)^{\prime}=0
$$

Hence if we choose

$$
p^{3}=p^{3}(r, t)
$$

we have according to $(4.17 \mathrm{~b})$

$$
Y_{2}=\partial_{2} Y, \quad Y_{3}=\partial_{3} Y
$$

where $Y$ is an arbitrary function of the coordinates. Therefore $(4.17 \mathrm{a}, \mathrm{c})$ yield

$$
\begin{aligned}
& \partial_{3} Y_{1}=\partial_{1} \partial_{3} Y-2\left(S_{343} \int p^{3} d t\right)^{\prime} \\
& \partial_{2} Y_{1}=\partial_{1} \partial_{2} Y-2 S_{242}^{\prime} p^{2} t .
\end{aligned}
$$

The integrability conditions of (4.20) reduce to

$$
\frac{\partial}{\partial \theta}\left(S_{343} \int p^{3} d t\right)^{\prime}=0
$$


and are satisfied because of (4.18). Hence the system (4.20) is completely integrable. The equations (4.14) are satisfied by

a)

b)

$$
\begin{gathered}
X_{\lambda}\left(X_{1}, X_{2}, X_{3}, 0\right) \\
p^{\nu}\left(0, \frac{a}{\sin \theta}, p^{3}(r, t), 0\right),
\end{gathered}
$$

where $X_{1}, X_{2}, X_{3}$ are given by (4.16), $Y_{2}, Y_{3}$ are given by (4.19), and $Y_{1}$ is the solution of the completely integrable system (4.20). In other words, (4.10) (with (4.21b)) satisfy the conditions of Theorem (4.4), so that (4.3) is another solution of (1.4b) and (1.5). The existence of the tensor $T_{\lambda \mu}{ }^{\nu}$ found in this illustration proves also the last statement of Theorem (4.4).

5. Continuation. A transformation

$$
\Gamma_{\lambda \mu}^{\nu}={ }^{0} \Gamma_{\lambda \mu}^{\nu}+\delta_{\lambda}^{\nu} p_{\mu}+\delta_{\lambda}^{\nu} p_{\lambda}
$$

(with an arbitrary vector $p_{\mu}$ ) does not affect the autoparallel lines (e.g. both connections have the same autoparallel lines). Therefore it is called an affine transformation. Moreover, both connections have the same skew-symmetric part and therefore (5.1) is called a symmetric transformation*. In this section we will apply the previous results on the transformations (5.1).

Theorem (5.1). Let ${ }^{0} \Gamma_{\lambda_{\mu}}^{\nu}$ be a solution of (1.4b) and (1.5). Let $\Gamma_{\lambda_{\mu}}^{\nu}$ be a connection with the following properties:

a) It has the same autoparallel lines as ${ }^{0} \Gamma_{\lambda \mu}^{\nu}$.

b) It has the same skew-symmetric part as ${ }^{0} \Gamma_{\lambda_{\mu}}^{\nu}$.

c) It is a solution of (1.4b) and (1.5) in the general case.

Then $\Gamma_{\lambda \mu}^{\nu}$ is given by (5.1) where the vector $p_{\lambda}$ satisfies the conditions

(5.2) a) ${ }^{0} D_{(\mu} p_{\lambda)}-p_{\mu} p_{\lambda}=0$

b) $\quad{ }^{0} S_{\lambda \mu}{ }^{\alpha} p_{\alpha}=\partial_{[\mu} Z_{\lambda]}$

with a suitably chosen vector $Z_{\lambda}$. The set of all vectors $p_{\lambda}$ satisfying (5.2) is not an empty set.

Proof. Every transformation of ${ }^{0} \Gamma_{\lambda \mu}^{\nu}$ may be written in the form (4.3). Requirement a) of our theorem is satisfied if and only if

$$
T_{(\lambda \mu)}^{\nu}=\delta_{\lambda}^{\nu} p_{\mu}+\delta_{\mu}^{\nu} p_{\lambda} .
$$

Requirement $b$ ) is satisfied if and only if

$$
T_{[\lambda \mu]}^{\nu}=0
$$

Hence we have

$$
T_{\lambda \mu}{ }^{\nu}=T_{(\lambda \mu)}^{\nu}=\delta_{\Lambda}^{\nu} p_{\mu}+\delta_{\lambda}^{\nu} p_{\lambda}, \quad T_{\lambda}=5 p_{\lambda},
$$

\footnotetext{
* Incidentally, (5.1) is the most general affine symmetric transformation.
} 
and consequently

$$
\begin{aligned}
{ }^{0} D_{\mu} T_{\lambda} & =5{ }^{0} D_{\mu} p_{\lambda} \\
2{ }^{0} S_{\alpha \mu}{ }^{\beta} T_{\lambda \beta}{ }^{\alpha} & =2{ }^{0} S_{\lambda \mu}{ }^{\alpha} p_{\alpha} \\
T_{\beta \mu}{ }^{\alpha} T_{\lambda \alpha}{ }^{\beta} & =7 p_{\mu} p_{\lambda} \\
-\left({ }^{0} D_{\alpha} T_{\lambda \mu}{ }^{\alpha}+T_{\alpha} T_{\lambda \mu}{ }^{\alpha}\right) & =-2\left({ }^{0} D_{(\lambda} p_{\mu)}+5 p_{\lambda} p_{\mu}\right) .
\end{aligned}
$$

Condition $(4.4 \mathrm{~b})$ reduces to

$$
4^{0} D_{\mu} p_{\lambda}-{ }^{0} D_{\lambda} p_{\mu}-3 p_{\mu} p_{\lambda}+2 S_{\lambda \mu}{ }^{\alpha} p_{\alpha}=\partial_{[\mu} X_{\lambda]}
$$

and may be split into two:

$$
\begin{gathered}
3^{0} D_{(\mu} p_{\lambda)}-3 p_{\mu} p_{\lambda}=0 \\
\text { b) } \quad 5^{0} D_{[\mu} p_{\lambda]}+2{S_{\lambda \mu}{ }^{\alpha} p_{\alpha}}=\partial_{[\mu} X_{\lambda]} .
\end{gathered}
$$

The equation (5.6a) is equivalent to (5.2a). Equation (5.6b) may be simplified to

$$
5 \partial_{[\mu} p_{\lambda]}-3{ }^{0} S_{\lambda \mu}{ }^{\alpha} p_{\alpha}=\partial_{[\mu} X_{\lambda]}
$$

This equation may be satisfied if and only if (5.2b) holds. The condition (4.9) is satisfied by virtue of (5.4). The last part of the theorem will be proved by the following

Illustration (5.1). Let us start again with the solution obtained in Section I 9 and put

$$
p_{\lambda}\left(0,0,0, p_{4}\right) .
$$

According to (5.7) and $\mathrm{I}(9.7)$ we have

$$
{ }^{0} S_{\lambda \mu}{ }^{\alpha} p_{\alpha}={ }^{0} S_{\lambda \mu}{ }^{4} p_{4}=0,
$$

so that (5.2b) is satisfied by a gradient $Z_{\lambda}=\partial_{\lambda} Z$. On the other hand we have by virtue of $\mathrm{I}(9.9), \mathrm{I}(9.14)$ and (5.7)

$$
{ }^{0} D_{(\mu} p_{\lambda)}=\delta_{(\lambda}^{4} \partial_{\mu)} p_{4}-\Gamma_{(\mu \lambda)}^{4} p_{4}=\delta_{(\lambda}^{4} \partial_{\mu)} p_{4}
$$

so that $(5.2 \mathrm{a})$ reduces to

$$
\delta_{(\lambda}^{4} \partial_{\mu)} p_{4}-\left(p_{4}\right)^{2} \delta_{\lambda}^{4} \delta_{\mu}^{4}=0
$$

and yields the solution

$$
p_{4}=\frac{1}{a-t}, \quad(a=\text { const. }) .
$$


Hence

$$
p_{\lambda}\left(0,0,0, \frac{1}{a-t}\right)
$$

satisfies the conditions (5.2) and (5.1) is another solution of (1.5) and (1.4b).

6. The most general solutions of (1.4) and (1.5). In previous sections we found the most general solution $\Gamma_{\lambda \mu}^{\nu}$ of $(1.4 \mathrm{~b})$ and (1.5) (by means of a particular solution $\left.{ }^{0} \Gamma_{\lambda \mu}^{\nu}\right)$. Applying then to $\Gamma_{\lambda \mu}^{\nu}$ the method of Section 3 we could find the corresponding tensor $g_{\lambda \mu}$ as a solution of (1.4a). Sometimes it is advisable to express $g_{\lambda \mu}$ by means of the tensor ${ }^{0} g_{\lambda \mu}$ (belonging to ${ }^{0} \Gamma_{\lambda_{\mu}}^{\nu}{ }^{*}$ ). In this present section we shall deal with this problem (and subsequent considerations). To start with, we return to the equation $I(1.9)$ and write

$$
{ }^{0} \Gamma_{\lambda \mu}^{\nu}=\left\{\begin{array}{c}
\nu \\
\lambda \mu
\end{array}\right\}+{ }^{0} S_{\lambda \mu}{ }^{\nu}+{ }^{0} U^{\nu}{ }_{\lambda \mu}
$$

Here $\left\{\begin{array}{c}\nu \\ \lambda \mu\end{array}\right\}$ are Christoffel symbols belonging to ${ }^{0} h_{\lambda \mu}={ }^{0} g_{(\lambda \mu)}$ while ${ }^{0} S_{\lambda \mu}{ }^{\nu},{ }^{0} U^{\nu}{ }_{\lambda \mu}$ are defined by equations similar to $\mathrm{I}(1.10)$ and $\mathrm{I}(1.11)$. Moreover, we denote by $\Gamma_{\lambda \mu}^{\nu}$ and $g_{\lambda \mu}$ another solution of (1.4) and (1.5). The connections ${ }^{0} \Gamma_{\lambda \mu}^{\nu}$ and $\Gamma_{\lambda \mu}^{\nu}$ are related by (4.3).

Theorem (6.1). The tensors $S_{\lambda \mu}{ }^{\nu}$ and ${ }^{0} S_{\lambda \mu}{ }^{\nu}, U^{\nu}{ }_{\lambda \mu}$ and ${ }^{0} U^{\nu}{ }_{\lambda \mu}$ are related by

a)

$$
S_{\lambda \mu}{ }^{\nu}={ }^{0} S_{\lambda \mu}{ }^{\nu}+T_{[\lambda \mu]}{ }^{\nu}
$$

b)

$$
U_{\lambda \mu}^{\nu}=\left\{\begin{array}{c}
\nu \\
\lambda \mu
\end{array}\right\}-\left\{\begin{array}{c}
\nu \\
\lambda \mu
\end{array}\right\}+T_{(\lambda \mu)}^{\nu}+{ }^{0} U_{\lambda \mu}^{\nu}
$$

The proof follows at once from

$$
\Gamma_{\lambda \mu}^{\nu}=\left\{\begin{array}{c}
\nu \\
\lambda \mu
\end{array}\right\}+{ }^{0} S_{\lambda \mu}{ }^{\nu}+{ }^{0} U^{\nu}{ }_{\lambda \mu}+T_{\lambda \mu}{ }^{\nu}=\left\{\begin{array}{c}
\nu \\
\lambda \mu
\end{array}\right\}+S_{\lambda \mu}{ }^{\nu}+U^{\nu}{ }_{\lambda \mu} .
$$

Note. This theorem is very efficient for a special kind of investigation. In the next theorem we shall give an illustration of its usefulness.

Theorem (6.2). Let ${ }^{0} g_{\lambda \mu},{ }^{0} \Gamma_{\lambda \mu}^{\nu}$ be a solution of (1.4) and (1.5). A necessary condition for the existence of another solution $g_{\lambda \mu}, \Gamma_{\lambda \mu}^{\nu}$ of (1.4) and (1.5) with the properties
(6.3) a) $\quad h_{\lambda \mu}={ }^{0} h_{\lambda \mu}$
b) $\quad S_{\lambda \mu}{ }^{\nu}={ }^{0} S_{\lambda \mu}{ }^{\nu}$,
c) $U_{\lambda \mu}^{\nu}=0$

\footnotetext{
* In Section I 9 we exhibited such a tensor ${ }^{0} g_{\lambda \mu}$ belonging to ${ }^{0} \Gamma_{\lambda \mu}^{\nu}$.
} 
is: the tensor ${ }^{0} U^{\nu}{ }_{\lambda \mu}$ satisfies the conditions

$$
\begin{aligned}
\partial_{[\mu} X_{\lambda]}={ }^{0} D_{\alpha}{ }^{0} U^{\alpha}{ }_{\lambda \mu} & +{ }^{0} U^{\alpha}{ }_{\beta(\mu}{ }^{0} U^{\beta}{ }_{\lambda) \alpha} \\
& -\left({ }^{0} D_{\mu}{ }^{0} U_{\lambda}+2{ }^{0} S_{\alpha \mu}{ }^{\beta}{ }^{0} U^{\alpha}{ }_{\lambda \beta}+{ }^{0} U_{\alpha}{ }^{0} U^{\alpha}{ }_{\lambda \mu}\right) \quad\left({ }^{0} U_{\mu} \equiv{ }^{0} U^{\alpha}{ }_{\alpha \mu}\right)
\end{aligned}
$$

where $X_{\mu}$ is an arbitrary vector (arbitrary gradient) in the general (special) case. ${ }^{*}$

Proof. If (6.3a) holds then

$$
\left\{\begin{array}{c}
\nu \\
\lambda \mu
\end{array}\right\}=\left\{\begin{array}{c}
\nu \\
\lambda \mu
\end{array}\right\} .
$$

If $(6.3 \mathrm{~b})$ holds, then by virtue of $(6.2 \mathrm{a})$

$$
T_{[\lambda \mu]}^{\nu}=0
$$

If (6.3c) holds then by virtue of $(6.2 \mathrm{~b})$ and $(6.5)$

$$
-T_{\lambda \mu}{ }^{\nu}={ }^{0} U^{\nu}{ }_{\lambda \mu}
$$

On the other hand

$$
U_{\beta \mu}^{\alpha} U_{\lambda \alpha}^{\beta}=U_{\beta(\mu}^{\alpha} U_{\lambda) \alpha}^{\alpha} \text {. }
$$

Now applying the Theorem (4.4) on $T_{\lambda \mu}{ }^{\nu}$ as given by (6.6) one obtains (6.4) by virtue of (6.7).

In the next theorem we use the following abbreviations

b)

$$
\begin{aligned}
& { }^{0} T_{\omega \lambda \mu}{ }^{\alpha \beta} \equiv T_{\lambda \omega}{ }^{\alpha} \delta_{\mu}^{\beta}+\delta_{\lambda}^{\alpha}\left(T_{\omega \mu}^{\beta}+2{ }^{0} S_{\omega \mu}^{\beta}\right), \\
& { }^{0} G_{\omega \lambda \mu} \equiv{ }^{0} g_{\alpha \beta}\left(T_{\omega \lambda \mu}{ }^{\alpha \beta}-2{ }^{0} S_{\omega \mu}{ }^{\beta} \delta_{\lambda}^{\alpha}\right) .
\end{aligned}
$$

Theorem (6.3). Denote by ${ }^{0} g_{\lambda \mu},{ }^{0} \Gamma_{\lambda_{\mu}}^{\nu}$ an arbitrary solution of (1.4) and (1.5) $\dagger$. Denote by $\Gamma_{\lambda \mu}^{\nu}$ (as given by (4.3)) a solution of (1.4b) and (1.5). In order that there exists a tensor $g_{\lambda \mu} \neq{ }^{0} g_{\lambda \mu}$ which (with the $\Gamma_{\lambda \mu}^{\nu}$ 's) is a solution of (1.4) and (1.5), it is necessary and sufficient that the system

$$
{ }^{0} D_{\omega} X_{\lambda \mu}=X_{\alpha \beta}{ }^{0} T_{\omega \wedge \mu}^{\alpha \beta}+{ }^{0} G_{\omega \lambda \mu}
$$

admit a solution $X_{\lambda \mu}$. If this condition is satisfied then

$$
g_{\lambda \mu}={ }^{0} g_{\lambda \mu}+X_{\lambda \mu} .
$$

\footnotetext{
* This vector is not necessarily the same as $X_{\mu}$ involved in (1.5).

† For instance the solution derived in Section $I 9$.
} 
Proof. First of all we have for $g_{\lambda \mu}$ (as given by (6.10)) according to (4.3) and (6.2a)

$$
\begin{aligned}
D_{\omega} g_{\lambda \mu} & =D_{\omega}\left({ }^{0} g_{\lambda \mu}+X_{\lambda \mu}\right) \\
& ={ }^{0} D_{\omega}\left({ }^{0} g_{\lambda \mu}+X_{\lambda \mu}\right)-T_{\lambda \omega}{ }^{\alpha}\left({ }^{0} g_{\alpha \mu}+X_{\alpha \mu}\right)-T_{\mu \omega}{ }^{\alpha}\left({ }^{0} g_{\lambda \alpha}+X_{\lambda \alpha}\right) \\
2 S_{\omega \mu}{ }^{\alpha} g_{\lambda \alpha} & =2\left[{ }^{0} S_{\omega \mu}{ }^{\beta}\left({ }^{0} g_{\lambda \beta}+X_{\lambda \beta}\right)+T_{[\omega \mu]}{ }^{\beta}\left({ }^{0} g_{\lambda \beta}+X_{\lambda \beta}\right)\right] .
\end{aligned}
$$

On the other hand we have

$$
{ }^{0} D_{\omega}{ }^{0} g_{\lambda \mu}=2{ }^{0} S_{\omega \mu}{ }^{\beta}{ }^{0} g_{\lambda \beta}
$$

A necessary and sufficient condition that $g_{\lambda \mu}$ be a solution of (1.4) and (1.5) (with the $\Gamma_{\lambda \mu}^{\nu}$ which are solutions of $(1.4 \mathrm{~b})$ and (1.5)) is

$$
D_{\omega} g_{\lambda \mu}=2 S_{\omega \mu}^{\beta} g_{\lambda \beta}
$$

Substituting into (6.12b) from (6.11) and remembering (6.12a) and (6.8), one obtains (6.9).

In order to formulate the main theorem of this chapter we introduce first the following tensors*:

a) ${ }^{0} A_{\xi \omega \lambda \mu}^{\alpha \beta} \equiv \frac{1}{2}^{0} R_{\xi \omega \lambda}{ }^{\alpha} \delta_{\mu}^{\beta}+\frac{1}{2} \delta_{\lambda}^{\alpha}{ }^{0} R_{\xi \omega \mu}{ }^{\beta}$

$$
-{ }^{0} S_{\omega \xi}{ }^{\gamma}{ }^{0} T_{\gamma \lambda \mu}^{\alpha \beta}-\delta_{[\xi}{ }^{0} T_{\omega] \lambda \mu}^{\gamma \delta}{ }^{0} T_{\nu \gamma \delta}^{\alpha \beta}-{ }^{0} D_{[\xi}{ }^{0} T_{\omega] \lambda \mu}^{\alpha \beta}
$$

b) $\quad{ }^{0} A_{\xi_{p+1} \cdots \xi_{1} \omega \lambda \mu}^{\alpha \beta} \equiv{ }^{0} D_{\xi_{p+1}}{ }^{0} A_{\xi_{p} \cdots \xi_{1} \omega \lambda \mu}^{\alpha \beta}+{ }^{0} T_{\xi_{p+1} \gamma \delta}^{\alpha \beta}{ }^{0} A_{\xi_{p} \cdots \xi_{1} \omega \lambda \mu}^{\gamma \delta}$

and

c) ${ }^{0} B_{\xi \omega \lambda \mu} \equiv{ }^{0} G_{\alpha \gamma \delta}\left({ }^{0} S_{\omega \xi}^{\alpha} \delta_{\lambda \mu}^{\gamma \delta}+\delta_{[\xi}^{\alpha}{ }^{0} T_{\omega] \lambda \mu}^{\gamma \delta}\right)$

d) ${ }^{0} B_{\xi_{p+1} \cdots \xi_{1} \omega \lambda \mu} \equiv{ }^{0} D_{\xi_{p+1}}{ }^{0} B_{\xi_{p} \cdots \xi_{1} \omega \lambda \mu}-{ }^{0} G_{\xi_{p+1} \gamma \delta}{ }^{0} A_{\xi_{p} \cdots \xi_{1} \omega \lambda \mu}^{\gamma \delta} . \quad(p=1,2, \cdots)$

Moreover, we denote by $\left(\left({ }^{0} A\right)\right)_{p}$ the matrix of 16 columns whose elements are ${ }^{0} A_{\xi_{p} \cdots \xi_{1} \omega \lambda \mu}^{\alpha \beta}$. The columns are labeled by superscripts $\alpha \beta$ while the rows are labeled by the subscripts $\xi_{p} \ldots \xi_{1} \omega \lambda \mu \dagger$. Another matrix used in the theorem is the matrix of 17 columns, denoted by $\left(\left({ }^{0} A \mid{ }^{0} B\right)\right)_{p}$. One obtains it by enlarging $((A))_{p}$ by one column, whose elements are ${ }^{0} B_{\xi_{p}} \cdots \xi_{1} \omega \lambda \mu$.

Theorem (6.4). Let ${ }^{0} g_{\lambda \mu}$ by a particular solution of (1.4) and (1.5)ł. Necessary

\footnotetext{
* The label 0 refers always to tensor constructed by means of ${ }^{0} \Gamma_{\lambda \mu}^{\nu}$. Thus for instance ${ }^{0} R_{\omega \mu \lambda}{ }^{\nu}$ is the curvature tensor of ${ }^{0} \Gamma_{\lambda \mu}^{\nu}$, and so on.

$\dagger$ The set of the first $4^{4}$ rows are labeled by $\xi_{1} \omega \lambda \mu$, the next set of $4^{5}$ rows are labeled by $\xi_{2} \xi_{1} \omega \lambda \mu$, and so on.

$\ddagger$ For instance the solution obtained in the Section I 9 .
} 
and sufficient conditions for the existence of another solution $g_{\lambda \mu}$ of (1.4) and (1.5) are: there is a tensor $T_{\lambda \mu}{ }^{\nu}$ with the following properties:

a) It satisfies (4.4b) and (4.9)*.

b) There is such an integer $N \geqq 1 \dagger$ that for any integer $p \geqq N$ the matrices $((A))_{p}$ and $((A \mid B))_{p}$ have the same rank $r \leqq 16$.

Whenever these conditions are satisfied then the system (6.9) is completely integrable, its solution contains 16 - $r$ arbitrary constants and satisfies the algebraic system

$$
X_{\alpha \beta}{ }^{0} A_{\xi_{q} \cdots \xi_{1} \omega \lambda \mu}^{\alpha \beta}={ }^{0} B_{\xi_{q} \cdots \xi_{1} \omega \lambda \mu} \quad(q=1, \cdots N) .
$$

The tensor $g_{\lambda \mu}$ is then given by (6.10), while the corresponding connection $\Gamma_{\lambda \mu}^{\nu}$ is given by (4.3). The set of all tensors $T_{\lambda \mu}{ }^{\nu}$ satisfying the conditions $\left.\left.\mathrm{a}\right), \mathrm{b}\right)$ is not an empty set.

Proof. The requirement a) has been found as necessary and sufficient for the connection (4.3) to be a solution of (1.4b) and (1.5). The equations (6.9) are necessary and sufficient for the existence of the corresponding tensor (6.10). Hence in order to complete the proof of Theorem (6.4) we have to find the integrability conditions for (6.9). The first set of these conditions is to be obtained from

$$
{ }^{0} D_{[\xi}{ }^{0} D_{\omega]} X_{\lambda \mu}={ }^{0} D_{[\xi}\left({ }^{0} T_{\omega] \lambda \mu}^{\alpha \beta} X_{\alpha \beta}+{ }^{0} G_{\omega] \lambda \mu}\right),
$$

where the covariant derivatives of $X_{\lambda \mu}$ are to be eliminated by means of (6.9) as well as by means of the well known identity of the type $I(6.2)$. In doing so, we obtain (6.14) for $q=1$. Taking the covariant derivative of this equation and eliminating $D_{\alpha} X_{\beta \gamma}$ by means of (6.9) we obtain (6.14) for $q=1,2$. Repeating this operation we obtain an infinite set of equations (6.14) for all $q=1,2, \cdots$. Then all the remaining statements (but the last one) of Theorem (6.4) are immediate consequences of the well known existence theorem $\ddagger$.

In order to prove the last statement we remember that the solution derived in the Section I 9 contains two arbitrary constants ( $c f r$. the equations I(9.16)). Hence, a suitable choice of these constants yields two solutions, one denoted by ${ }^{0} g_{\lambda \mu}$, the other by $g_{\lambda \mu}$. Then the equations (6.9) admit the solutions $X_{\lambda \mu}=$ $g_{\lambda \mu}-{ }^{0} g_{\lambda \mu}$ so that the requirements of Theorem (6.4) must be satisfied.

Corollary (6.1). Whenever $r=16$ then the solution $X_{\lambda \mu}$ of (6.9) may be obtained in a purely algebraic way, by solving the system (6.14).

The proof follows easily from the previous theorem.

7. Particular cases. In this section we shall deal with particular cases of the field $g_{\lambda \mu}$ in connection with $U^{\nu}{ }_{\lambda \mu}$. The first case is characterized by

$$
U^{\nu}{ }_{\lambda \mu}=0
$$

\footnotetext{
* The connection ${ }^{0} \Gamma_{\lambda \mu}^{\nu}$ involved in (4.4b) belongs to ${ }^{0} g_{\lambda \mu}$ as a solution of (1.4).

$\dagger$ We have obviously $N \leqq 16$.

$\ddagger C f r$. for instance [3] p. 17 .
} 
and needed later on. We know (cfr. Theorem (9.1) in [6]) that (7.1) is a necessary and sufficient condition for the connections $\Gamma_{\lambda \mu}^{\nu}$ and $\left\{\begin{array}{c}\nu \\ \lambda \mu\end{array}\right\}$ to have the same paths (i.e. the same autoparallel lines).

Theorem (7.1). Necessary and sufficient conditions for a solution $g_{\lambda_{\mu}}$ of (1.4) and (1.5) to satisfy (7.1) are

a)

c)

$$
\begin{aligned}
{k_{(\rho}{ }^{\alpha} K_{\omega) \mu \alpha}} & =0, \\
H_{\mu \lambda} & =\frac{1}{4} K_{\alpha \mu}{ }^{\beta} K_{\beta \lambda}{ }^{\alpha}, \\
\nabla_{\alpha} K_{\mu \lambda}{ }^{\alpha} & =2 \partial_{[\mu} X_{\lambda]},
\end{aligned}
$$

where $X_{\lambda}$ is an arbitrary vector (arbitrary gradient) in the general (in the special) case.

Proof. Equation (7.2a) is a necessary and sufficient condition for the solution $\Gamma_{\lambda \mu}^{\nu}$ of (1.4a) to satisfy (7.1) (cfr. Theorems (9.1) and (9.2a) in [6]). Assume now that for a solution $\Gamma_{\lambda \mu}^{\nu}$ of $(1.4 \mathrm{a}, \mathrm{b})$ the condition $(7.2 \mathrm{a})$ holds, so that (7.1) is satisfied. Then the tensor $B_{\lambda \mu}$ as defined by $\mathrm{I}(7.6)$ is equal to zero, and consequently $\mathrm{I}(7.5)$ reduces to

$$
R_{(\mu \lambda)}=H_{\mu \lambda}-\frac{1}{4} K_{\alpha \mu}^{\beta} K_{\beta \lambda}^{\alpha} .
$$

Moreover, the tensor $A_{\mu \lambda}$ as defined by I(7.4) vanishes by virtue of (7.1), and consequently the condition $(1.4 \mathrm{~b})$ reduces $\mathrm{I}(7.2 \mathrm{~b})$ to

$$
R_{[\mu \lambda]}=\frac{1}{2} \nabla_{\alpha} K_{\mu \lambda}{ }^{\alpha} .
$$

Hence, we obtain (7.2b, c) from (7.3) and (1.5). Assume now that the condition (7.1) holds for a solution $\Gamma_{\lambda \mu}^{\nu}$ of $(1.4 a, b)$. Then the same method yields (7.2).

Theorem (7.2). The set of all tensors $g_{\lambda \mu}$ satisfying (1.4) and $(7.2)^{*}$ is not an empty set.

Proof. Let us consider the tensors $k_{\lambda \mu}$ and $h_{\lambda \mu}$ as given by $\mathrm{I}(8.6 \mathrm{a})$ and $\mathrm{I}(8.8)$. Then we have first**

$$
\frac{1}{2} \nabla_{[\mu} k_{\lambda v]}=\partial_{[\mu} E_{\lambda} u_{\nu]}=E_{[\lambda} \partial_{\mu} u_{\nu]}=E_{[\lambda} E_{\mu} u_{\nu]}^{\prime}=0
$$

so that according to $\mathrm{I}(1.12)$

$$
K_{\mu \lambda \nu}=2 \nabla_{\nu} k_{\mu \lambda}=4 \partial_{\nu} E_{[\mu} u_{\lambda]}=4 E_{[\mu} u_{\lambda]}^{\prime} E_{\nu} .
$$

\footnotetext{
* We have to bear in mind that $(7.2 \mathrm{~b}, \mathrm{c})$ are equivalent to (1.4b) and (1.5) in our case.

** The prime indicates the derivative with respect to $Z$.
} 
Consequently

$$
\begin{aligned}
\frac{1}{4} K_{\alpha \mu}^{\beta} K_{\beta \lambda}^{\alpha} & =4 E^{\beta} E^{\alpha} E_{[\alpha} u_{\mu]}^{\prime} E_{[\beta} u_{\lambda]}^{\prime} \\
& =\left(E^{\alpha} u_{\alpha}^{\prime}\right)^{2} E_{\mu} E_{\lambda}=0 \\
\nabla_{\alpha} K_{\mu \lambda}^{\alpha} & =4 \partial_{\alpha} E^{\alpha} E_{[\mu} u_{\lambda]}^{\prime}=4 E^{\alpha} \partial_{\alpha} E_{[\mu} u_{\lambda]}^{\prime} \\
& =4 E^{\alpha}\left(\partial_{\alpha} u_{[\lambda}^{\prime}\right) E_{\mu]}=-4\left(\partial_{3} u_{[\lambda}^{\prime}+\partial_{4} u_{[\lambda}^{\prime}\right) E_{\mu]} \\
& =-4\left(u_{[\lambda}^{\prime \prime}-u_{[\lambda}^{\prime \prime}\right) E_{\mu]}=0 .
\end{aligned}
$$

On the other hand we have according to $\mathrm{I}(8.8 \mathrm{c})$

$$
H_{\mu \lambda}=0 .
$$

Hence, the equations (7.2) are satisfied for a gradient $X_{\lambda}$ and the proof follows easily.

In the last theorem of this section we will investigate the influence of $k=0^{*}$ on $U^{\nu}{ }_{\lambda \mu}$. Moreover, we assume that (1.4) are satisfied.

Theorem (7.3). When $k_{\lambda \mu}$ belongs to either the second or the third class** then

$$
3 U_{\alpha} k_{\omega}{ }^{\alpha}+U_{\alpha \beta \omega} k^{\beta \alpha}=0 .
$$

When $k_{\lambda \mu}$ belongs to the third class, then

$$
U_{\alpha \beta \omega} k^{\beta \alpha}=0 .
$$

Proof. The equation I(1.11b) yields by virtue of (1.4b)

$$
0=K_{\omega \alpha}{ }^{\alpha}-2 U_{\alpha \beta}{ }^{\beta} k_{\omega}{ }^{\alpha}+2 U_{\alpha \beta \omega} k^{\beta \alpha} .
$$

On the other hand we have from $\mathrm{I}(1.12)$ and $\mathrm{I}(1.10 \mathrm{c})$
(7.5) b)
$K_{\omega \alpha}{ }^{\alpha}=2 \nabla_{\alpha} k_{\omega}{ }^{\alpha}$,
c)
$2 U_{\alpha \beta}{ }^{\beta}=-4 U_{\alpha}$,

so that $(7.5 \mathrm{a})$ reduces to

$$
\nabla_{\alpha} k_{\omega}{ }^{\alpha}+2 U_{\alpha} k_{\omega}{ }^{\alpha}+U_{\alpha \beta \omega} k^{\beta \alpha}=0 .
$$

This equation holds regardless of $k$. If $k=0$ then we obtain from $\mathrm{I}(3.8 \mathrm{a})$

$$
m_{\lambda \nu}=-\frac{1}{2 \sqrt{g}} \mathfrak{e}_{\lambda \nu \alpha \beta} \sqrt{|\mathfrak{h}|} k^{\alpha \beta}
$$

\footnotetext{
$* k$ is defined by $\mathrm{I}(1.4)$.

** See the first paragraphi of the Section I 1.
} 
so that $I(5.3 \mathrm{~b}) *$ reduces to

$$
2 \sqrt{|\mathfrak{h}|} \nabla_{\alpha} k^{\omega \alpha}=-\sqrt{g} \mathbb{E}^{\omega \mu \lambda \nu} U_{\mu}\left(-\frac{1}{2 \sqrt{g}} e_{\lambda \nu \alpha \beta} \sqrt{|\mathfrak{h}|} k^{\alpha \beta}\right)=2 U_{\mu} k^{\omega \mu} \sqrt{|\mathfrak{h}|}
$$

or

$$
\nabla_{\alpha} k_{\omega}{ }^{\alpha}=U_{\alpha} k_{\omega}{ }^{\alpha} \text {. }
$$

Comparing (7.6a) and (7.6b) one obtains (7.4a). On the other hand if $k_{\lambda \mu}$ belongs either to the second or to the third class, then we have $k=0$. If $k_{\lambda \mu}$ belongs to the third class, then we have not only $k=0$ but also $k_{\alpha \beta} k^{\alpha \beta}=0$, so that according to $\mathrm{I}(1.5)$ we have $g=1$ and $\mathrm{I}(1.13 \mathrm{~b})$ yields

$$
U_{\lambda}=0 \text {. }
$$

Comparing this equation with (7.4a) one obtains (7.4b).

In three subsequent papers we shall deal with applications of results obtained in these two chapters.

\section{BIBLIOGRAPHY}

[1] Einstein, Albert, The Meaning of Relativity, 3rd ed., Princeton, 1950, pp. 162.

[2] Einstein, Albert, The Meaning of Relativity, 4th ed., Princeton, 1953.

[3] Eisenhart, L. P., Non-Riemannian Geometry, Am. Math. Soc., 1927, pp. 179.

[4] HlavatÝ, V., "The Elementary Basic Principles of the Unified Theory of Relativity," Proc. Nat. Acad. Sci., vol. 38, 1952, p. 243-247.

[5] Hlavatý, V., "The Elementary Basic Principles of the Unified Theory of Relativity. A," J. Rational Mech. Anal., vol. 1, 1952, p. 539-562.

[6] Hlavaty, V., "The Elementary Basic Principles of the Unified Theory of Relativity. B," J. Rational Mech. Anal., vol. 2, 1953, p. 1-52.

[7] HuavatÝ, V., \& SÁenz, A. W., "Uniqueness Theorems in the Unified Theory of Relativity," J. Rational Mech. Anal., vol. 2, 1953, p. 523-536.

* We already know that $I(5.2)$ is equivalent to $S_{\lambda}=0$. 